\title{
An Adapted Loss Function for Censored Quantile Regression
}

\author{
Mickaël De Backer* $\quad$ Anouar El Ghouch* Ingrid Van Keilegom,*
}

November 27, 2017

\begin{abstract}
In this paper, we study a novel approach for the estimation of quantiles when facing potential right censoring of the responses. Contrary to the existing literature on the subject, the adopted strategy of this paper is to tackle censoring at the very level of the loss function usually employed for the computation of quantiles, the so-called "check" function. For interpretation purposes, a simple comparison with the latter reveals how censoring is accounted for in the newly proposed loss function. Subsequently, when considering the inclusion of covariates for conditional quantile estimation, by defining a new general loss function the proposed methodology opens the gate to numerous parametric, semiparametric and nonparametric modelling techniques. In order to illustrate this statement, we consider the well-studied linear regression under the usual assumption of conditional independence between the true response and the censoring variable. For practical minimization of the studied loss function, we also provide a simple algorithmic procedure shown to yield satisfactory results for the proposed estimator with respect to the existing literature in an extensive simulation study. From a more theoretical prospect, consistency and asymptotic normality of the estimator for linear regression are obtained using several recent results on non-smooth semiparametric estimation equations with an infinite-dimensional nuisance parameter, while numerical examples illustrate the adequateness of a simple bootstrap procedure for inferential purposes. Lastly, an application to a real dataset is used to further illustrate the validity and finite sample performance of the proposed estimator.
\end{abstract}

Key words: Check function; Linear regression; Beran estimator; MM algorithm; Bootstrap

\footnotetext{
${ }^{*}$ Université catholique de Louvain, Institut de Statistique, Biostatistique et Sciences Actuarielles. Voie du Roman Pays 20, B-1348 Louvain-la-Neuve, Belgium. Corresponding e-mail: mickael.debacker@uclouvain.be.

${ }^{\dagger} \mathrm{KU}$ Leuven, Research Centre for Operations Research and Business Statistics. Naamsestraat 69, 3000 Leuven, Belgium.
} 


\section{Introduction}

Since the pioneering work of Koenker and Bassett (1978), quantile regression has become a preeminent substitute to the classical least-squares regression in both theoretical and applied statistics. While mean regression models solely grasp the central behavior of the data, quantile regression allows the analyst to investigate the complete distributional information of the dependence of the response variable on a set of one or more covariates at hand. In that sense, quantile regression represents a fundamental tool in applications where extremes are important, such as clinical trials or environmental studies where upper and lower quantiles levels are of essential concern. An interesting illustration of this may be found for instance in Elsner et al. (2008), where the average intensity of hurricanes is observed to be steady over the years while upper quantiles are shown to be increasing. This information should then crucially be accounted for in any proper risk or environmental model. Further advantages of quantile regression include, amongst others, flexibility to the error distribution and robustness to outlying observations. A book length treatment of the methodology may be found in Koenker (2005).

Existing literature on the estimation of a quantile regression function includes numerous methodologies for fully observed response observations. In practice however, many interesting applications are affected by possible right censoring of the responses due, for instance, to the withdrawal of patients in biomedical studies, or the end of the follow-up period of a clinical trial. As discussed in Koenker and Bilias (2001), Koenker and Geling (2001) and Portnoy (2003), when confronted to such data, quantile regression provides a valuable complement to the commonly used Cox proportional hazards model or the accelerated failure time model, with ease of interpretation, flexibility to possible heterogeneity in the data and robustness counting as primary benefits.

The introduction of censored quantile regression goes back to Powell $(1984 ; 1986)$ in the econometric literature for linear models and the particular case of fixed censoring, where it is assumed that the censoring variable is always observable. For random censoring, as it is mostly the case in survival analysis, the main rationale of the current literature has been so far to take censoring into account through the formulation of synthetic data points or weighting schemes. One such weighting scheme was introduced by Portnoy (2003), where the robustness of quantile regression is exploited through the concept of redistribution-of-mass first developed by Efron (1967). The underlying idea is to redistribute the mass of censored data lying under the quantile of interest to artificial outlying observations to the right, as the contribution of each point to the estimation of the quantile regression only depends on the sign of the residual. However, given that the weights to be redistributed are to be determined by the conditional distribution of the survival time given the covariates, Portnoy's estimation scheme was developed under a restrictive global linearity assumption to simplify the procedure, that is, assuming that the regression function is linear for all quantiles levels in $(0, \tau)$, where $\tau$ is the quantile level of interest. Relaxing this assumption by only assuming linearity at the level of interest, and exploiting the same idea of redistribution-ofmass, Wang and Wang (2009) proposed a locally weighted estimation scheme based on Beran's local Kaplan-Meier estimator (Beran (1981)) for the conditional distribution of the variable of interest instead of Portnoy's global assumption. Nonetheless, the handling of kernel smoothing causes the estimator of Wang and Wang to be unsuited for the handling of multiple and categorical covariates. This motivated Wey et al. (2014) to exploit, in the same methodology, survival trees instead of Beran's estimator for the required conditional distribution estimation. Overall, the redistribution-of-mass methodology proved to be a valuable approach for parametric censored quantile regression and lead to further research such as variable selection (Wang et al. (2013)), multiple quantile estimation (Tang and Wang (2015)) and cure rate quantile regression (Wu and Yin (2016)). However, it should be noted that this general weighting strategy relies on an appropriate estimation of the conditional distribution of the variable of interest. As a consequence, by nature, this strategy only seems appropriate for parametric models as any further extensions of the technique to semiparametric or nonparametric regressions would seem questionable given one would have to adopt a preliminary local estimation of the conditional distribution for, in fact, a 
local estimator of the conditional quantile.

An alternative popular weighting scheme in the literature is the so-called inverse-censoringprobability, which was adapted to the linear quantile regression through different versions by Ying et al. (1995), Bang and Tsiatis (2002) and Leng and Tong (2013) to cite a few. This technique is, in spirit, similar to the notorious mean regression estimator of Koul et al. (1981). The simplicity of use and its ease of interpretation engendered further research such as variable selection (Shows et al. (2010)) as well as extensions outside the linear area, such as nonparametric regression (e.g. El Ghouch and Van Keilegom (2009)), single-index regression (Bücher et al. (2014)) or copula-based regression (De Backer et al. (2017)) among others. This advantageous simplicity of adaptation of the technique to a nonlinear regression literature is to be contrasted with the redistribution-ofmass approach. However, major shortcomings of this weighting scheme are the need to evaluate a (possibly local) Kaplan-Meier estimator of the censoring distribution in the right tail, for which Zhou (2006) proposed a simple bypass taking the robustness of quantile regression into account, and, more constraining, an efficiency loss in the estimation as roughly only uncensored observations are preserved in the procedure.

Besides these fruitful weighting schemes, an interesting idea was proposed by Lindgren (1997) where, rather than studying weighting strategies for the observed data, the idea was to focus on the quantile level and convert the problem of finding the $\tau$-th conditional quantile of the unobserved variable of interest into finding a superior quantile of the actual observed responses. However, no asymptotics were given and the involved iterative procedure lacks theoretical background.

In this paper, we aim at providing a new insight into censored quantile regression by tackling the problem of censoring through an alternative strategy to the above-discussed literature. In fact, instead of handling weighting schemes or substitute quantile levels, we propose to account for censoring at the very level of the loss function used in quantile regression, the so-called "check" function. Intuitively, when confronted to right censored datasets, the proposed loss function will penalize more severely underestimation of the value of the regression than the usual check function. The methodology then allows to plainly exploit the information of all the observations at hand, hence avoiding any estimation efficiency loss. However, as a possible inconvenience, the studied loss function is observed to be no longer convex, which then requires the proposal of an efficient algorithmic procedure to appropriately minimize the resulting objective function. In this paper, a simple adjustment of the Majorize-Minimize (MM) algorithm as proposed by Hunter and Lange (2000) is therefore examined. Apart from this, it is worth stressing out that by concentrating on a censored version of the check function, the proposed strategy has the potential to engender multiple extensions to the linear context considered here. The latter could for instance concern parametric models and variable selection in penalized regression, but one could also easily accommodate the strategy to nonparametric and semiparametric models. Therefore, the overall objective of this paper is to illustrate this novel general estimation strategy in a simple and well-studied linear context in order to analyze its behavior in comparison to the existing literature, and hence guide any potential further research on the advantages and pitfalls of this strategy.

The rest of this paper is organised as follows. Motivating the origin of the newly proposed loss function first for sample quantiles, and then illustrating its accommodation for linear regression is the topic of Section 2. Investigating further the linear context, consistency and asymptotic normality of the proposed estimator are obtained in Section 3. Section 4 provides a detailed adaptation of the MM algorithm to practically implement the procedure, and the finite sample performance of the latter is illustrated by means of Monte Carlo simulations in Section 5. Section 6 highlights a brief application to real data. Lastly, the proofs of Section 3 are deferred to the Appendix. 


\section{The Proposed Methodology}

\subsection{Quantiles without covariates}

To motivate and illustrate the proposed methodology, we introduce in this section a simple onedimensional example and consider the problem of finding the $\tau$-th quantile $m_{\tau}$ of a survival time variable $T$, or some transformation of the latter. For any $\tau \in(0,1)$, the $\tau$-th quantile is defined as $m_{\tau}=\inf \left\{t: F_{T}(t) \geq \tau\right\}$, where $F_{T}$ is the continuous cumulative distribution function (c.d.f.) of $T$. From the seminal work of Koenker and Bassett (1978), it is well-known that this problem is equivalent to solving

$$
m_{\tau}=\arg \min _{a} \mathbb{E}\left[\rho_{\tau}(a ; T)\right],
$$

where $\rho_{\tau}(a ; T)=(T-a)(\tau-\mathbb{1}(T \leq a))$ is the so-called "check" function, and $\mathbb{1}(\cdot)$ is the indicator function.

Let us suppose now that the variable of interest $T$ is subject to censoring, that is, instead of observing $T$, one only observes $(Y, \Delta)$, where $Y=\min (T, C), \Delta=\mathbb{1}(T \leq C)$ and $C$ denotes the censoring variable assumed for now to be independent of $T$, with c.d.f. $G_{C}$. In this context, finding $m_{\tau}$ is equivalent to finding the root in $a$ of the equation

$$
\mathbb{E}\left[\mathbb{1}(Y>a)-\bar{G}_{C}(a)(1-\tau)\right]=0,
$$

with $\bar{G}_{C}(a):=1-G_{C}(a)=\mathbb{P}(C>a)$. For this equivalence to hold, it is required that $T$ is independent of $C$, and that $\bar{G}_{C}\left(m_{\tau}\right)>0$. Note that this latter condition, routinely made in survival analysis, also amounts to establishing a natural upper bound for the quantile of interest that can be studied in the presence of censored data.

Now, starting from (2.2), mimicking the reasoning behind the check function $\rho_{\tau}$ leads us to define the following function in $a$, for a given $y \in \mathbb{R}$ and $G_{C}$ :

$$
\varphi_{\tau}\left(a ; y, G_{C}\right)=\rho_{\tau}(a ; y)-(1-\tau) \int_{0}^{a} G_{C}(s) \mathrm{d} s,
$$

which is equal to $\int_{a}^{y}\left(\mathbb{1}(y>s)-\bar{G}_{C}(s)(1-\tau)\right) \mathrm{d} s$, up to a constant with respect to $a$. Our claim is that the function $\varphi_{\tau}$ actually is an extended version of the check function $\rho_{\tau}$, such that the effect of censoring is handled at the very level of the loss function through a correcting term $(1-\tau) \int_{0}^{a} G_{C}(s) \mathrm{d} s$. To support this statement, first note that when all observations are complete, the above-defined function trivially boils down to the check function. Next, observe that, for all values of $a \neq y, \partial \varphi_{\tau}\left(a ; y, G_{C}\right) / \partial a=\bar{G}_{C}(a)(1-\tau)-\mathbb{1}(y>a)$. Since $0 \leq \bar{G}_{C}(a) \leq 1, \forall a$, this suggests that for a value $y<\tau_{G_{C}}=\inf \left\{t: G_{C}(t)=1\right\}, a \mapsto \varphi_{\tau}\left(a ; y, G_{C}\right)$ is strictly decreasing on $(-\infty, y)$, strictly increasing on $\left[y, \tau_{G_{C}}\right)$, and hence with global minimum value at $a=y$, exactly as the check function. Now, searching for the minimum value in $a$ of $\mathbb{E}\left[\varphi_{\tau}\left(a ; Y, G_{C}\right)\right]$ reveals to be corresponding to the formulation of (2.2), as

$$
\frac{\partial}{\partial a} \mathbb{E}\left[\varphi_{\tau}\left(a ; Y, G_{C}\right)\right]=\bar{G}_{C}(a)(1-\tau)-\mathbb{P}(Y>a) .
$$

From these observations, one may conclude that $\varphi_{\tau}$ is an adapted version of the check function $\rho_{\tau}$ accounting for potential incompleteness of observations. Therefore, in the presence of censoring, we define the logical counterpart of (2.1):

$$
m_{\tau}=\arg \min _{a} \mathbb{E}\left[\varphi_{\tau}\left(a ; Y, G_{C}\right)\right] .
$$

Accordingly, based on an i.i.d. sample $\left(Y_{i}, \Delta_{i}\right), i=1, \ldots, n$, of $(Y, \Delta)$, and given an estimator $\widehat{G}_{C}$ of $G_{C}$, we define a natural estimator of $m_{\tau}$ as the empirical version of (2.3):

$$
\widehat{m}_{\tau}=\arg \min _{a} \sum_{i=1}^{n} \varphi_{\tau}\left(a ; Y_{i}, \widehat{G}_{C}\right) .
$$



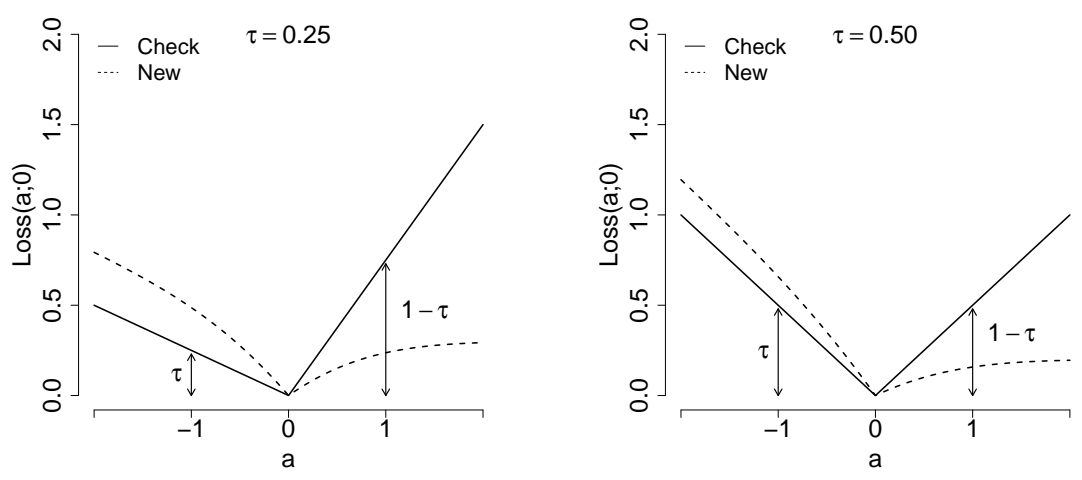

Figure 1: Comparison of the check function $a \mapsto \rho_{\tau}(a ; 0)$ with the censored loss function $a \mapsto \varphi_{\tau}\left(a ; 0, G_{C}\right)$ for $\tau=\{0.25,0.5\}$ and $C \sim \mathcal{N}(0,1)$.

Note that $\hat{m}_{\tau}$ exploits all observations in the estimation process, regardless of their censoring status. From the above-described reasoning, it also naturally appears that this general loss function may then be extended outside basic sample quantiles to numerous regression models, as will be illustrated next for the linear model.

To gain further insight into the newly proposed loss function and understand how censoring is accounted for through the regulating term $(1-\tau) \int_{0}^{a} G_{C}(s) \mathrm{d} s$, we consider in Figure 1 a graphical illustration for the situation $y=0$ with two quantile levels $\tau=\{0.25,0.5\}$ and a standard normal distribution for the censoring variable. Note that in this situation, the correcting term of $\varphi_{\tau}$ will always be positive for $a>0$, while negative for $a<0$. Intuitively, this simply suggests that $\varphi_{\tau}$ penalizes more heavily underestimation of the value of the quantile than the usual check function to correct for the fact that the response may not be fully observed. Similarly, as can be graphically observed, a smaller loss is assigned for overestimation in order to stimulate the latter when using incomplete data. Note also that the penalization will logically be impacted by the distribution $G_{C}$, as the amount of alteration of the loss function will be all the more important given that $G_{C}\left(m_{\tau}\right)=\mathbb{P}\left(C \leq m_{\tau}\right)$ is large. This is in line with one's intuition that censoring should especially be corrected for when it is susceptible of altering the estimation of a quantile, that is, when censoring is likely to happen to data points lying below $m_{\tau}$.

Finally, an important feature that should be noted, appearing clearly in Figure 1, is that $a \mapsto \varphi_{\tau}\left(a ; y, G_{C}\right)$ is in fact no longer convex. Therefore, special care is called for the numerical optimization required in (2.4) as the objective function may allow for local minima, hereby possibly harming the search for the global one. In light of this, we provide in Section 4 an adapted version of the Majorize-Minimize (MM) algorithm as proposed by Hunter and Lange (2000) that proves to be satisfactory regarding the simulation results of Section 5 , even in more complex multivariate regression situations.

\subsection{Quantiles with covariates: linear regression}

To apply the newly proposed loss function in a regression context, we consider the well-studied linear regression model. Specifically, we consider the inclusion of a covariate vector $\boldsymbol{X}$ of dimension $d+1, d \geq 1$, whose first component corresponding to the intercept is taken to be 1 . Then, based on an i.i.d. sample $\left(T_{i}, \boldsymbol{X}_{i}\right), i=1, \ldots, n$, from $(T, \boldsymbol{X})$, it is assumed that

$$
m_{\tau}\left(\boldsymbol{X}_{i}\right)=\beta_{\tau}^{\top} \boldsymbol{X}_{i},
$$

where $m_{\tau}(\boldsymbol{x})=\inf \left\{t: F_{T \mid \boldsymbol{X}}(t \mid \boldsymbol{x}) \geq \tau\right\}$ is the $\tau$-th conditional quantile of $T$ given $\boldsymbol{X}=\boldsymbol{x}, F_{T \mid \boldsymbol{X}}$ denotes the conditional c.d.f. of $T$ given $\boldsymbol{X}$, and $\beta_{\tau}$ is the $(d+1)$-dimensional unknown quantile 
coefficient vector. Similarly to sample quantiles, it is well known that the estimation of $\beta_{\tau}$ in the uncensored case is obtained by minimizing the objective function

$$
Q_{n}(\beta)=\sum_{i=1}^{n} \rho_{\tau}\left(\beta^{\top} \boldsymbol{X}_{i} ; T_{i}\right)
$$

Under random censoring, the statistical problem now consists of estimating the true value of $\beta_{\tau}$ based on i.i.d triplets $\left(Y_{i}, \Delta_{i}, \boldsymbol{X}_{i}\right), i=1, \ldots, n$, from $(Y, \Delta, \boldsymbol{X})$, where, as in Section 2.1, $Y=\min (T, C), \Delta=\mathbb{1}(T \leq C)$ and $C$ is assumed here to be independent of $T$ given $\boldsymbol{X}$. In this context, analogous arguments to those previously described lead us to define $\beta_{\tau}$ as the minimizer of the function

$$
Q^{c}(\beta)=\mathbb{E}\left[\varphi_{\tau}\left(\beta^{\top} \boldsymbol{X} ; Y, G_{C}(\cdot \mid \boldsymbol{X})\right)\right],
$$

where the superscript $c$ explicitly expresses the presence of censoring, and where $G_{C}(\cdot \mid \boldsymbol{x})$ denotes the conditional distribution of $C$ given $\boldsymbol{X}=\boldsymbol{x}$. Subsequently, we propose to estimate $\beta_{\tau}$ by minimizing the following objective function:

$$
Q_{n}^{c}(\beta)=\sum_{i=1}^{n} \varphi_{\tau}\left(\beta^{\top} \boldsymbol{X}_{i} ; Y_{i}, \widehat{G}_{C}\left(\cdot \mid \boldsymbol{X}_{i}\right)\right)=\sum_{i=1}^{n}\left\{\rho_{\tau}\left(\beta^{\top} \boldsymbol{X}_{i} ; Y_{i}\right)-(1-\tau) \int_{0}^{\beta^{\top} \boldsymbol{X}_{i}} \widehat{G}_{C}\left(s \mid \boldsymbol{X}_{i}\right) \mathrm{d} s\right\},
$$

where $\widehat{G}_{C}(\cdot \mid \boldsymbol{x})$ is a consistent estimator of $G_{C}(\cdot \mid \boldsymbol{x})$. Popular choices for the latter include, among others, the Kaplan-Meier estimator if one assumes independence between $C$ and $\boldsymbol{X}$, a Cox regression model or Beran's prominent conditional Kaplan-Meier estimator. The latter takes the following form for Nadaraya-Watson type of weights:

$$
\widehat{G}_{C}(c \mid \boldsymbol{x})=1-\prod_{i=1}^{n}\left\{1-\frac{B_{n i}(\boldsymbol{x})}{\sum_{j=1}^{n} \mathbb{1}\left(Y_{i} \leq Y_{j}\right) B_{n j}(\boldsymbol{x})}\right\}^{\mathbb{1}\left(Y_{i} \leq c, \Delta_{i}=0\right)},
$$

where $B_{n j}(\boldsymbol{x})=K\left(\left(\boldsymbol{x}-\boldsymbol{X}_{j}\right) / h_{n}\right) / \sum_{k=1}^{n} K\left(\left(\boldsymbol{x}-\boldsymbol{X}_{k}\right) / h_{n}\right)$ is a sequence of weights depending on some multivariate kernel density function $K$ and a positive bandwidth parameter $h_{n}$.

Similarly to sample quantiles, formulation (2.7) allows one to plainly extract the information of every observation at hand, even if confronted to incompleteness of the latter. In comparison, the initial inverse-censoring-probability strategy of Koul et al. (1981) has to trade-off between appropriate estimation of $G_{C}$ and efficiency loss through the use of $\Delta$ in the adopted weights. Therefore, one could expect that the proposed methodology will be more efficient than competitors constructed on the basic inverse-censoring-probability strategy, especially when the censoring proportion is large. In the same spirit, one might expect that the above-described estimator provides in this linear context an interesting alternative to the redistribution-of-mass approach when confronted to high censoring percentages, as the latter will inevitably suffer from low sample size for the required estimation of $F_{T \mid \boldsymbol{X}}(\cdot \mid \boldsymbol{x})$ in this situation. However, a possible drawback of the formulation (2.7) compared to its competitors is that the estimation of $G_{C}(\cdot \mid \boldsymbol{x})$ will affect in our context all observations, hereby possibly harming the global estimation process in case of low sample size for the estimation of the latter distribution. These preliminary remarks will be empirically investigated in Section 5.

\section{Large Sample Properties}

We establish in this section both the consistency and asymptotic normality of the proposed estimator $\widehat{\beta}_{\tau}$ defined as the minimizer of $Q_{n}^{c}(\beta)$ in (2.7). To that end, we require that the following assumptions hold:

(C1) The support $\operatorname{supp}(\boldsymbol{X})$ of $\boldsymbol{X}$ is contained in a compact subset of $\mathbb{R}^{d+1}$, and the variancecovariance matrix of $\boldsymbol{X}$ is positive definite. 
(C2) For $\beta$ in a neighborhood $\mathcal{B}$ of $\beta_{\tau}$, $\inf _{\boldsymbol{x} \in \operatorname{supp}(\boldsymbol{X})} f_{T \mid \boldsymbol{X}}\left(\beta^{\top} \boldsymbol{x} \mid \boldsymbol{x}\right)>0$, where $f_{T \mid \boldsymbol{X}}(\cdot \mid \boldsymbol{x})$ denotes the conditional density function of $T$ given $\boldsymbol{X}=\boldsymbol{x}$. Furthermore, $\sup _{t, \boldsymbol{x} \in \operatorname{supp}(\boldsymbol{X})} f_{T \mid \boldsymbol{X}}(t \mid \boldsymbol{x})<\infty$.

(C3) Define the (possibly infinite) time $\tau_{F_{Y}(\cdot \mid \boldsymbol{x})}=\inf \left\{t: F_{Y \mid \boldsymbol{X}}(t \mid \boldsymbol{x})=1\right\}$, where $F_{Y \mid \boldsymbol{X}}$ designates the conditional c.d.f. of $Y$ given $\boldsymbol{X}$. Suppose first that there exists a real number $v<$ $\tau_{F_{Y}(\cdot \mid \boldsymbol{x})}$ for all $\boldsymbol{x}$ in $\operatorname{supp}(\boldsymbol{X})$. Denote next by $\mathcal{G}$ the class of functions $\left.\left.G(t, \boldsymbol{x}):\right]-\infty, v\right] \times$ $\operatorname{supp}(\boldsymbol{X}) \rightarrow[0,1]$ of bounded variation with respect to $t$ (uniformly in $\boldsymbol{x}$ ) that have first-order partial derivatives with respect to $\boldsymbol{x}$ of bounded variation in $t$ (uniformly in $\boldsymbol{x}$ ), and bounded (uniformly in $t$ ) second-order partial derivatives with respect to $\boldsymbol{x}$ which are uniformly in $t$ Lipschitz of order $\eta$ for some $0<\eta<1$. Suppose that $G_{C} \in \mathcal{G}$.

(C4) For every $\boldsymbol{x} \in \operatorname{supp}(\boldsymbol{X})$ and for $\beta \in \mathcal{B}$, the point $\beta^{\top} \boldsymbol{x} \in \mathbb{R}$ lies below $v$.

(C5) The estimator $\hat{G}_{C}$ of $G_{C}$ satisfies:

(i) $\sup _{\boldsymbol{x} \in \operatorname{supp}(\boldsymbol{X})} \sup _{y \leq v}\left|\widehat{G}_{C}(y \mid \boldsymbol{x})-G_{C}(y \mid \boldsymbol{x})\right|=o_{\mathbb{P}}(1)$ and $\mathbb{P}\left(\widehat{G}_{C} \in \mathcal{G}\right) \rightarrow 1$ as $n \rightarrow \infty$.

(ii) $\mathbb{E}_{\boldsymbol{X}}\left[\boldsymbol{X}\left(\widehat{G}_{C}\left(\beta^{\top} \boldsymbol{X} \mid \boldsymbol{X}\right)-G_{C}\left(\beta^{\top} \boldsymbol{X} \mid \boldsymbol{X}\right)\right)\right]=n^{-1} \sum_{i=1}^{n} \boldsymbol{X}_{i} \xi\left(Y_{i}, \Delta_{i}, \beta^{\top} \boldsymbol{X}_{i} \mid \boldsymbol{X}_{i}\right)+o_{\mathbb{P}}\left(n^{-1 / 2}\right)$, uniformly in $\beta \in \mathcal{B}$, where the notation $\mathbb{E}_{\boldsymbol{X}}$ explicitly indicates that the expectation is taken with respect to the distribution of $\boldsymbol{X}$, and where $\xi\left(Y_{i}, \Delta_{i}, \beta^{\top} \boldsymbol{X}_{i} \mid \boldsymbol{X}_{i}\right), i=1, \ldots, n$, are i.i.d. random vectors with mean 0 and $\sup _{\beta \in \mathcal{B}} \mathbb{E}\left[\left\|\xi\left(Y_{i}, \Delta_{i}, \beta^{\top} \boldsymbol{X}_{i} \mid \boldsymbol{X}_{i}\right)\right\|^{2}\right]<\infty$.

Before commenting our main assumptions, it should be noted that our proofs both rely on a crucial result of Lopez (2011) for the class $\mathcal{G}$ defined in assumption (C3). The latter result is constructed upon a dimension reduction technique of the form $G_{C}(\cdot \mid \boldsymbol{X})=G_{C}(\cdot \mid g(\boldsymbol{X}))$ for some function $g: \mathbb{R}^{d+1} \rightarrow \mathbb{R}$. As a consequence, our proofs are here valid as such under the same hypothesis or, more simply, for univariate covariates.

Now, assumptions $(\mathrm{C} 1)$ and $(\mathrm{C} 2)$ are standard in the context of quantile regression estimation for both complete and censored observations in order namely to guarantee the uniqueness of $\beta_{\tau}$. Assumption (C3) defines a general class of functions embedding $G_{C}$ coming from the work of Lopez (2011). The latter paper develops the theory of bracketing numbers (defined in Van der Vaart and Wellner (1996, p. 83)) associated to the class $\mathcal{G}$ on which part of the proofs rely. Assumption (C4) is required for both the uniqueness of $\beta_{\tau}$ and the asymptotic properties of $\hat{\beta}_{\tau}$. Note that (C4) also encompasses the remark following equation (2.2) in Section 2.1, as it defines here in the regression context a natural upper bound for the quantile of interest that can be studied when considering censored responses. Assumption (C5)-(i) requires in its first part the uniform consistency of the censoring distribution estimator and is for instance fulfilled with a conditional, respectively unconditional, Kaplan-Meier estimator as shown in Van Keilegom and Akritas (1999), respectively Gill (1983), under namely suitable bandwidth conditions for the former. The second part of (C5)-(i) may also be verified using for instance a conditional Kaplan-Meier estimator under appropriate bandwidth and kernel conditions as stated in Lemma 6.2 of Lopez (2011). Finally, assumption (C5)-(ii) is requested for the asymptotic distribution of our estimator and requires implicitly a general linear representation of $\widehat{G}_{C}$. Stating such an assumption allows us not to be restrictive for the estimator one wishes to consider for $G_{C}$. It should of course be noted that, when selecting a particular form of estimator, such an assumption can in fact be deduced from appropriate higher-level conditions. To illustrate this statement, Lemma 1 in the Appendix exposes how condition (C5)-(ii) may be deduced from bandwidth and kernel conditions if one adopts Beran's estimator as described in (2.8). A similar development for the appropriate transcription of (C5)-(ii) in the case of a Kaplan-Meier estimator for $G_{C}$ may follow the exact same arguments along with the linear representation proposed in Lo and Singh (1986).

We now state in Theorem 3.1 the consistency of our proposed estimator. Its proof is deferred to the Appendix. 
Theorem 3.1. For a given quantile level $0<\tau<1$, define $\widehat{\beta}_{\tau}$ as the minimizer of $Q_{n}^{c}(\beta)$ in $(2.7)$. Assume that the censoring time $C$ is conditionally independent of the survival time $T$ given the covariates $\boldsymbol{X}$, and that the triples $\left(Y_{i}, \Delta_{i}, \boldsymbol{X}_{i}\right), i=1, \ldots, n$, form an i.i.d. multivariate random sample. Then, under assumptions (C1)-(C5)-(i),

$$
\hat{\beta}_{\tau} \rightarrow \beta_{\tau}
$$

in probability, as $n \rightarrow \infty$.

The proof of Theorem 3.1 relies heavily on the work of Delsol and Van Keilegom (2015) on non-smooth semiparametric estimating equations with an infinite-dimensional nuisance parameter. The first key requirement to apply this work to our framework is the uniform consistency of the conditional censoring distribution estimator. The second key requirement concerns the class $\mathcal{G}$ for which it has to be shown that the latter is 'well-behaved' in terms of size, which is represented by the notion of bracketing number, in order for $\widehat{\beta}_{\tau}$ to be consistent when constructed on a preliminary estimator $\hat{G}_{C} \in \mathcal{G}$. For this part, we rely on a crucial result of Lopez (2011) which establishes a bound on bracketing numbers for the class $\mathcal{G}$ under the above-mentioned dimension reduction technique.

Next, we report in the following theorem the limiting distribution of our proposed estimator $\hat{\beta}_{\tau}$. For this result, we rely on Proposition 2 in Birke et al. (2017) which slightly modifies Theorem 2 of Chen et al. (2003), and where the key requirement is now the linear representation of the nuisance parameter implied by assumption (C5)-(ii).

Theorem 3.2. For a given $0<\tau<1$, under the assumptions of Theorem 3.1 and (C5)-(ii),

$$
n^{1 / 2}\left(\widehat{\beta}_{\tau}-\beta_{\tau}\right) \stackrel{\mathcal{L}}{\rightarrow} \mathcal{N}\left(0, \Gamma_{1}^{-1} \Sigma \Gamma_{1}^{-1}\right),
$$

where $\Gamma_{1}=\mathbb{E}\left[\boldsymbol{X} \boldsymbol{X}^{\top} f_{T \mid \boldsymbol{X}}\left(\beta_{\tau}^{\top} \boldsymbol{X} \mid \boldsymbol{X}\right)\left(1-G_{C}\left(\beta_{\tau}^{\top} \boldsymbol{X} \mid \boldsymbol{X}\right)\right)\right]$, and $\Sigma=\operatorname{Cov}\left(\Lambda_{i}\right)$ with $\Lambda_{i}$ defined in equation (A.5) of the Appendix.

Practical inferential use of Theorem 3.2 on $\beta_{\tau}$ is made difficult by the complex form of the asymptotic covariance matrix as the latter involves several unknown quantities that are challenging to accurately estimate in practice. Therefore, for inferential purposes we propose to adopt the simple percentile bootstrap through resampling the triples $\left(Y_{i}, \Delta_{i}, \boldsymbol{X}_{i}\right), i=1, \ldots, n$, with replacement. Specifically, $95 \%$ bootstrap confidence intervals for $\beta_{\tau}$ may easily be constructed using the 2.5th and 97.5th percentiles of the bootstrap coefficients, after having drawn a sufficient amount of bootstrap samples. This technique was repeatedly shown to be satisfactory in the literature (e.g. Portnoy (2003), Wang and Wang (2009), Leng and Tong (2013)) and its validity for the proposed procedure will be illustrated in Section 5 .

\section{Minimization Algorithm}

As discussed in Section 2, application of the newly proposed methodology for linear regression has to account for the computational aspect of the mathematical minimization of (2.7). In particular, in addition to the classical differentiability issue of quantile regression, the nonconvexity of $a \mapsto$ $\varphi_{\tau}\left(a ; Y_{i}, \widehat{G}_{C}\left(\cdot \mid \boldsymbol{X}_{i}\right)\right)$ requires an efficient optimization routine for the global minimization of the objective function. Given that the newly proposed loss function may be seen as an extension of the check function, we suggest in this section to adapt an existing methodology for uncensored data, for which much work has been provided for the linear context as current procedures include, amongst others, the Interior point algorithm, Simplex algorithms and the MM algorithm. We therefore first briefly review in this section one particular optimization procedure for complete observations, and then discuss how the latter may be adapted to the practical minimization of $(2.7)$. 
Primarily due to its numerical robustness and ease of adaptation, we propose to investigate in our context the use of the MM, or Majorize-Minimize, algorithm as first introduced by Hunter and Lange (2000) for quantile regression. As suggested by its denomination, the rationale of the MM algorithm is to operate in two steps: the objective function to be minimized is first majorized by an appropriate surrogate function, which is then in turn minimized in the second step in order to define the next iterate of the algorithm. By doing so, a difficult optimization problem is to be replaced by a simpler one, with iteration counting as the price to pay for this substitution. More formally, in the quantile regression context with complete observations, letting $\beta^{(m)}$ denote the $m$-th iterate in finding the minimum of $Q_{n}(\beta)$ defined in (2.6), Hunter and Lange propose in the first step to majorize $Q_{n}(\beta)$ by a surrogate function $\xi_{n}\left(\beta \mid \beta_{(m)}\right): \mathbb{R}^{(d+1)} \times \mathbb{R}^{(d+1)} \rightarrow \mathbb{R}$ such that $\xi_{n}\left(\beta \mid \beta_{(m)}\right) \geq Q_{n}(\beta)$, for all $\beta$ and $\xi_{n}\left(\beta_{(m)} \mid \beta_{(m)}\right)=Q_{n}\left(\beta_{(m)}\right)$. Specifically, exploiting the fact that majorization relations are closed under the formation of sums, Hunter and Lange suggest to majorize $Q_{n}(\beta)$ by the sum for each $i=1, \ldots, n$, of the unique quadratic curve tangent to the graph of $\rho_{\tau}\left(\beta_{(m)}^{\top} \boldsymbol{X}_{i} ; T_{i}\right)$ at $\beta_{(m)}^{\top} \boldsymbol{X}_{i}$, yielding

$$
\xi_{n}\left(\beta \mid \beta_{(m)}\right)=\sum_{i=1}^{n}\left\{\frac{\left(T_{i}-\beta^{\top} \boldsymbol{X}_{i}\right)^{2}}{4\left(\epsilon+\left|T_{i}-\beta_{(m)}^{\top} \boldsymbol{X}_{i}\right|\right)}+\left(\tau-\frac{1}{2}\right)\left(T_{i}-\beta^{\top} \boldsymbol{X}_{i}\right)\right\}+c_{\tau},
$$

where $c_{\tau}$ is a constant such that $\xi_{n}\left(\beta_{(m)} \mid \beta_{(m)}\right)=Q_{n}\left(\beta_{(m)}\right)$, and $\epsilon>0$ is a small perturbation to be selected in order to avoid issues with possible zero residuals for iteration $m$. The explicit minimizer of $\xi_{n}\left(\beta \mid \beta_{(m)}\right)$ with respect to $\beta$ then simply becomes the next iterate $\beta_{(m+1)}$ in the second step of the MM algorithm. As an interesting property, called 'descent property', it can be shown that the MM algorithm automatically drives the objective function downhill, that is $\xi_{n}\left(\beta_{(m+1)} \mid \beta_{(m)}\right) \leq \xi_{n}\left(\beta_{(m)} \mid \beta_{(m)}\right)$.

Applying the same arguments with the objective of now finding the minimizer of $Q_{n}^{c}(\beta)$ defined in (2.7), we note that $a \mapsto \varphi_{\tau}\left(a ; Y_{i}, \widehat{G}_{C}\left(\cdot \mid \boldsymbol{X}_{i}\right)\right)$ is the difference of two convex functions. In the search of a surrogate function, this suggests then to explicitly use the quadratic form of $\xi_{n}\left(\beta \mid \beta_{(m)}\right)$ for the first part of $\varphi_{\tau}$, and exploit the convexity of the second part for which we have that for all $i=1, \ldots, n$,

$$
\int_{0}^{\beta^{\top} \boldsymbol{X}_{i}} \widehat{G}_{C}\left(s \mid \boldsymbol{X}_{i}\right) \mathrm{d} s \geq \int_{0}^{\beta_{(m)}^{\top} \boldsymbol{X}_{i}} \widehat{G}_{C}\left(s \mid \boldsymbol{X}_{i}\right) \mathrm{d} s+\left(\beta^{\top} \boldsymbol{X}_{i}-\beta_{(m)}^{\top} \boldsymbol{X}_{i}\right) \widehat{G}_{C}\left(\beta_{(m)}^{\top} \boldsymbol{X}_{i} \mid \boldsymbol{X}_{i}\right) .
$$

Recombining this with the expression of $\xi_{n}\left(\beta \mid \beta_{(m)}\right)$ yields the following surrogate function for $Q_{n}^{c}(\beta)$ :

$$
\begin{aligned}
\xi_{n}^{c}\left(\beta \mid \beta_{(m)}\right)= & \sum_{i=1}^{n}\left\{\frac{\left(Y_{i}-\beta^{\top} \boldsymbol{X}_{i}\right)^{2}}{4\left(\epsilon+\left|Y_{i}-\beta_{(m)}^{\top} \boldsymbol{X}_{i}\right|\right)}+\left(\tau-\frac{1}{2}\right)\left(Y_{i}-\beta^{\top} \boldsymbol{X}_{i}\right)\right. \\
& \left.-(1-\tau) \int_{0}^{\beta_{(m)}^{\top} \boldsymbol{X}_{i}} \widehat{G}_{C}\left(s \mid \boldsymbol{X}_{i}\right) \mathrm{d} s-(1-\tau)\left(\beta^{\top} \boldsymbol{X}_{i}-\beta_{(m)}^{\top} \boldsymbol{X}_{i}\right) \widehat{G}_{C}\left(\beta_{(m)}^{\boldsymbol{\top}} \boldsymbol{X}_{i} \mid \boldsymbol{X}_{i}\right)\right\}+\tilde{c}_{\tau},
\end{aligned}
$$

where $\tilde{c}_{\tau}$ is a constant such that $\xi_{n}^{c}\left(\beta_{(m)} \mid \beta_{(m)}\right)=Q_{n}^{c}\left(\beta_{(m)}\right)$. For the second step of the algorithm, let $\mathcal{X}=\left[\boldsymbol{X}_{1}, \ldots, \boldsymbol{X}_{n}\right]$ be the $(d+1) \times n$ matrix of covariates, and $\mathcal{Y}^{\top}=\left(Y_{1}, \ldots, Y_{n}\right)$ be the vector of observed responses. Minimizing $\xi_{n}^{c}\left(\beta \mid \beta_{(m)}\right)$ with respect to $\beta$ to obtain $\beta_{(m+1)}$ then yields the following succinct result:

$$
-\mathcal{X} \mathcal{A}_{(m)} \mathcal{Y}+\mathcal{X} \mathcal{A}_{(m)} \mathcal{X}^{\top} \beta_{(m+1)}-\mathcal{X D}=\mathcal{X} \mathcal{E}_{(m)},
$$

where $\mathcal{A}_{(m)}$ is a $n \times n$ diagonal matrix with $i$-th diagonal entry $1 /\left[2\left(\epsilon-\left|Y_{i}-\beta_{(m)}^{\top} \boldsymbol{X}_{i}\right|\right)\right], \mathcal{D}$ is a $n \times 1$ vector of $(\tau-1 / 2)$, and $\mathcal{E}_{(m)}$ is a $n \times 1$ vector with $i$-th entry $(1-\tau) \widehat{G}_{C}\left(\beta_{(m)}^{\top} \boldsymbol{X}_{i} \mid \boldsymbol{X}_{i}\right)$. Solving (4.1) with respect to $\beta_{(m+1)}$ yields the explicit iteration of the MM algorithm at step $(m+1)$, which is a simple adaptation of the MM algorithm for linear quantile regression with complete observations as there is only a supplementary term $\mathcal{E}_{(m)}$ appearing at every iteration. The proposed algorithm may then be resumed as follows: 


\section{Algorithm}

Step 0. Given an initial estimate of $\beta_{\tau}$ denoted by $\beta_{(0)}$, set $m=0$. Select a small tolerance value $\delta$, for instance $\delta=10^{-9}$, and choose $\epsilon$ such that $\epsilon \ln \epsilon \approx-\delta / n$.

Step 1. Estimate $G_{C}(\cdot \mid \boldsymbol{X})$ and calculate $\mathcal{E}_{(m)}$ as $(1-\tau) \widehat{G}_{C}\left(\beta_{(m)}^{\top} \boldsymbol{X}_{i} \mid \boldsymbol{X}_{i}\right), i=1, \ldots, n$. Calculate $\mathcal{A}_{(m)}$ and set

$$
\beta_{(m+1)}=\left(\mathcal{X} \mathcal{A}_{(m)} \mathcal{X}^{\top}\right)^{-1} \mathcal{X}\left(\mathcal{A}_{(m)} \mathcal{Y}+\mathcal{D}+\mathcal{E}_{(m)}\right)
$$

Step 2. If ||$\beta_{(m+1)}-\beta_{(m)}||>\delta$ or $\left|\xi_{n}^{c}\left(\beta_{(m+1)} \mid \beta_{(m)}\right)-\xi_{n}^{c}\left(\beta_{(m)} \mid \beta_{(m)}\right)\right|>\delta$, replace $m$ by $m+1$ and return to step 1 .

Concerning the choice of $\beta_{(0)}$, special care is to be brought to the latter given the nonconvexity of $\varphi_{\tau}$, and hence the possibility of capturing only a local minimum, if there is any. In an attempt to mend this obstacle, similarly to Leng and Tong (2013), we propose to adopt the estimator of Bang and Tsiatis (2002) as $\beta_{(0)}$, given the consistency of the latter. Hence, one could hope that, by already providing a descent estimation of $\beta_{\tau}, \beta_{(0)}$ should be close to the global minimum of $Q_{n}^{c}(\beta)$, hereby possibly avoiding the ambush of potential local minima. Furthermore, given the need at each iteration $m$ of the algorithm to evaluate $\widehat{G}_{C}\left(\beta_{(m)}^{\top} \boldsymbol{X}_{i} \mid \boldsymbol{X}_{i}\right), i=1, \ldots, n$, a descent estimator $\beta_{(0)}$ may also, to some extent, prevent too many evaluations of $\widehat{G}_{C}(\cdot \mid \boldsymbol{X})$ in the right tail, for which classical estimators are known to be typically unstable due to sparsity of the data. On the other hand, as noted by Leng and Tong, given that only uncensored observations are handled for $\beta_{(0)}$, the latter estimator may severely suffer from an efficiency loss in the estimation, hereby motivating the use of an estimator exploiting all observations at hand such as the one proposed in this paper. Nonetheless, as there is no guarantee that the algorithm starting from this $\beta_{(0)}$ will avoid converging to a possible local minimum, it is preferable to restart the latter as suggested by Hunter and Lange, in this case for instance by adding small perturbations to $\beta_{(0)}$ to verify the stability of the solution.

Finally, note that, analogously to the remarks of Section 2, the described algorithmic procedure of this section based on the MM algorithm is again easily adaptable to broader parametric, nonparametric and semiparametric models. For instance, considering a general parametric model, a convenient minimization algorithmic procedure would only require an adaptation of equation (4.1) to define the appropriate iterative process one could readily implement for the desired model.

\section{Simulation Study}

In this section, we assess the finite sample performance of the proposed methodology by means of Monte Carlo simulations. As previously mentioned, given that the adapted loss function may engender further modelling techniques beyond the simple linear framework, we are mainly interested here in comparing the performance of our estimator (NEW) with the two prominent weighting schemes for quantile regression with censored data: the redistribution-of-mass (RM), which will be embodied here by Wang and Wang's estimator, and the inverse-censoring-probability (ICP), represented here by Bang and Tsiatis' estimator. Based on the choice of these competitors, as a preliminary remark that has repeatedly been illustrated in the literature, while ICP may be subject to possible improvement for linear models, RM provides in this context a very competitive estimator, often taken as primary reference. Lastly, to grasp the impact of censoring, we also include an omniscient procedure (Omni) using all the observations $T_{i}, i=1, \ldots, n$, as if they were available in practice for the estimation of $\beta_{\tau}$.

The four procedures are compared in four main data generating processes (DGP) where the survival time is systematically independent of the censoring time given the covariates. The first three DGPs are taken to be univariate settings, while the fourth setting considers an example with four covariates. Given that both RM and NEW will be implemented with local estimators 
of conditional distributions as will be detailed below, the latter setting will be of interest in order to analyze the performance of both procedures when confronted to multivariate covariates. Note that, in their paper on variable selection using the redistribution-of-mass, Wang et al. (2013) also tolerate as many as four covariates for the determination of the involved local weights.

The first DGP is taken from Wang and Wang (2009) and Leng and Tong (2013), and presents a simple setting which is linear in all quantile levels and where all estimation procedures in the literature perform relatively well for the estimation of the regression coefficients. Hence, a basic requirement for a newly proposed methodology as the one considered here would be to perform appropriately as well in this particular setting. Next, the second and third DGP, partly inspired by Wang and Wang, are taken to be linear only in the quantile level of interest, hereby ruling out the use of estimators assuming global linearity such as Portnoy's or the martingale-based estimator of Peng and Huang (2008), despite their relative robustness illustrated for instance in Wang and Wang and Leng and Tong. For the second DGP, the censoring variable $C$ is taken to be independent of the covariate, while the third DGP covers a dependent scenario. This will allow to explore the potential impact of a conditional censoring distribution. Note however that, in all four settings, the proposed procedure NEW will repeatedly be implemented considering a conditional censoring distribution even when the simulated settings do not present any influence of the covariate on the latter distribution.

Concerning the implementation of these procedures, we first use the rq function of the R library quantreg for Omni. ICP is likewise implemented with rq by incorporating in the function the weights $\Delta_{i} /\left(1-\widehat{G}_{C}\left(Y_{i}\right)\right), i=1, \ldots, n$, where $\widehat{G}_{C}$ is the Kaplan-Meier estimator of $G_{C}$. As for $\mathrm{RM}$, the estimator is implemented using Wang and Wang's code, available on their websites. For the estimation of local weights, we use the biquadratic kernel $K(x)=(15 / 16)\left(1-x^{2}\right)^{2} \mathbb{1}(|x| \leq 1)$ for the univariate settings as recommended in Wang and Wang, while an eighth-order kernel $K(x)=(1 / 13)\left(1-x^{2}\right)\left(35-385 x^{2}+1001 x^{4}-715 x^{6}\right) \mathbb{1}(|x| \leq 1)$ is used for the four-variate example, just as in Wang et al.. For the first three settings, the bandwidth of the procedure is determined by 5 -fold cross validation among 15 candidates equally ranging from 0.05 to 0.5 , whereas one initial simulated dataset serves for the determination of the bandwidth for the multivariate DGP among 15 candidates equally ranging from 0.5 to 2 given the computational cost of searching for tuning parameters across multiple dimensions. Lastly, NEW is implemented using the algorithm of Section 4 where, for every DGP we adopt Beran's local Kaplan-Meier estimator as $\widehat{G}_{C}(\cdot \mid \boldsymbol{X})$. As for RM, the latter is based either on the biquadratic or the eighth-order kernel depending on the dimension of the covariate. Finally, the required bandwidth is likewise computed via 5-fold cross validation at each iteration or on one initial dataset. This suggests that for the last DGP, the performance of both RM and NEW could probably be improved if the bandwidths were adapted to each simulation, although none of the estimators is here favored over the other in the analysis we intend to provide.

\section{DGP 1.}

The following model is generated:

$$
T_{i}=\beta_{0}+\beta_{1} X_{i}+\eta_{i}
$$

where $\beta_{0}=3, \beta_{1}=5, X_{1}, \ldots, X_{n}$ are i.i.d. $U[0,1]$ variables and $\eta_{1}, \ldots, \eta_{n}$ are i.i.d. $\mathcal{N}(0,1)$ variables. The censoring variables $C_{1}, \ldots, C_{n}$ are simulated from $U[0, M]$, with $M$ calibrated to attain the desired censoring proportion $(15 \%$ or $40 \%)$ at the median. We note that the choice of $M$ also implies that condition (C4) is not violated for the true $\beta_{\tau}$, where $\tau=0.5$ in this case. The latter consideration regarding condition $(\mathrm{C} 4)$ repeatedly applies to all the following DGPs and for all the considered levels of $\tau$. 


\section{DGP 2.}

The following model is generated:

$$
T_{i}=\beta_{0}+\beta_{1} X_{i}+\left(3+\left(X_{i}-0.5\right)^{2}\right)\left(\eta_{i}-\Phi^{-1}(\tau)\right),
$$

where $\beta_{0}=1, \beta_{1}=0.1, X_{1}, \ldots, X_{n}$ are i.i.d. $\mathcal{N}(0,1)$ variables, $\eta_{1}, \ldots, \eta_{n}$ are also i.i.d. $\mathcal{N}(0,1)$ variables and $\Phi^{-1}$ denotes the quantile function of the standard normal distribution. The censoring variables $C_{1}, \ldots, C_{n}$ are simulated from $U[m, M]$, where $m=-5 / 3$ for $\tau=0.3, m=-3$ for $\tau=0.5$, $m=-3.5$ for $\tau=0.7$, and $M$ is chosen to attain the desired censoring proportions ( $30 \%$ and $60 \%$ ) depending on the considered quantile levels.

\section{DGP 3.}

The model to generate $T_{i}, i=1, \ldots, n$, is the same as for DGP 2 but with parameters $\beta_{0}=1$ and $\beta_{1}=1$. Additionally, in this setting the censoring variable is generated from the model $C_{i}=\gamma_{0}+\gamma_{1} X_{i}+v_{i}, i=1, \ldots, n$, where $\gamma_{0}=1, \gamma_{1}=1$, and $v_{1}, \ldots, v_{n}$ are i.i.d. variables from $U[m, M]$, where $m=-2$ for $30 \%$ censoring, $m=-4$ for $60 \%$ censoring, and $M$ is calibrated to obtain the desired censoring proportion for at the median.

\section{DGP 4.}

The following model, inspired by Shows et al. (2010), is generated:

$$
T_{i}=\beta_{0}+\beta_{1} X_{1 i}+\beta_{2} X_{2 i}+\beta_{3} X_{3 i}+\beta_{4} X_{4 i}+\eta_{i},
$$

where $\beta_{0}=1, \beta_{1}=0.5, \beta_{2}=1, \beta_{3}=1.5, \beta_{4}=2, X_{j i}$ are i.i.d. standard normal variables for $i=1, \ldots, n$, and $j=1, \ldots, 4$, and $\eta_{1}, \ldots, \eta_{n}$, are i.i.d. variables simulated from $t(5)$. The variables $C_{1}, \ldots, C_{n}$ are, here again, simulated from $U[m, M]$, where $m=0$ for $30 \%$ censoring, $m=-2$ for $60 \%$ censoring, and $M$ is chosen to obtain the desired censoring proportion for $\tau=0.5$.

For all simulation settings, we consider $B=500$ repetitions of each DGP, four average censoring proportions $\left(p_{c} \in\{15 \%, 40 \%\}\right.$ for DGP 1 and $p_{c} \in\{30 \%, 60 \%\}$ for DGP 2, 3 and 4$)$, and sample sizes $n \in\{100,200,500\}$. The quantile levels of interest are chosen among $\{0.3,0.5,0.7\}$ depending on the DGP and the censoring proportion of interest. Estimators are compared in terms of bias, root mean squared errors (RMSE), and median absolute error (MAE). We further include an overall criterion taken from a prediction point of view: the mean absolute deviation (MAD), given for an estimator $\widehat{\beta}$ by $n^{-1} \sum_{i=1}^{n}\left|\widehat{\beta}^{\top} \boldsymbol{X}_{i}-\beta_{\tau}^{\top} \boldsymbol{X}_{i}\right|$.

Table 1 reports the results of our simulation study for DGP 1 . As expected, all estimation procedures perform very similarly in this basic setting, although ICP already exhibits some relative difficulties to compete when confronted to higher censoring proportions. Concerning NEW, we note that in this example the procedure is relatively robust to the low sample size for computing the required local Kaplan-Meier estimator, as observed for $n=100$ and $p_{c}=15 \%$. Furthermore, even though the simulated scenario does not account for any influence of the covariate on the censoring distribution, our simulation experience with the present DGP suggests that the estimator is relatively robust to the smoothing parameter to be selected when using Beran's estimator $\widehat{G}_{C}(\cdot \mid X)$. This robustness of the results towards both the sample size and the smoothing parameter may be considered as an encouraging observation for the newly proposed estimator given that all observations are affected by the conditional censoring distribution estimator through the studied adapted loss function as mentioned in Section 2.

Moving to a more challenging setting with higher censoring proportions, Table 2 reports the results of our simulation study for DGP 2 with $n=200$ observations at each iteration. Due to identifiability issues as encountered in condition (C4), the quantile level $\tau=0.7$ is not considered here when simulating as much as $60 \%$ censoring, whereas the quantile level $\tau=0.5$ is left aside for $30 \%$ censoring for the sake of brevity. From these results, we observe that both RM and ICP 


\begin{tabular}{|c|c|c|c|c|c|c|c|c|c|}
\hline \multirow[b]{2}{*}{$n$} & \multirow[b]{2}{*}{$p_{c}$} & \multirow[b]{2}{*}{ Method } & \multicolumn{2}{|c|}{ Bias } & \multicolumn{2}{|c|}{ RMSE } & \multicolumn{2}{|c|}{ MAE } & \multirow[b]{2}{*}{ MAD } \\
\hline & & & $\beta_{0}$ & $\beta_{1}$ & $\beta_{0}$ & $\beta_{1}$ & $\beta_{0}$ & $\beta_{1}$ & \\
\hline \multirow{8}{*}{100} & \multirow{4}{*}{$15 \%$} & Omni & 0.007 & 0.000 & 0.255 & 0.425 & $\begin{array}{c}0.168 \\
\end{array}$ & 0.281 & 0.135 \\
\hline & & NEW & 0.013 & -0.006 & 0.267 & 0.454 & 0.175 & 0.282 & 0.147 \\
\hline & & RM & 0.009 & -0.004 & 0.267 & 0.459 & 0.173 & 0.286 & 0.148 \\
\hline & & ICP & 0.010 & -0.006 & 0.270 & 0.456 & 0.174 & 0.283 & 0.148 \\
\hline & \multirow{4}{*}{$40 \%$} & Omni & 0.007 & 0.000 & 0.255 & 0.425 & 0.168 & 0.281 & 0.135 \\
\hline & & NEW & 0.009 & 0.013 & 0.298 & 0.558 & 0.183 & 0.343 & 0.177 \\
\hline & & RM & 0.001 & 0.003 & 0.297 & 0.552 & 0.184 & 0.361 & 0.176 \\
\hline & & ICP & 0.009 & -0.001 & 0.311 & 0.583 & 0.185 & 0.384 & 0.181 \\
\hline \multirow{8}{*}{200} & \multirow{4}{*}{$15 \%$} & $\overline{\overline{O m n i}}$ & $\overline{c 0.009}$ & -0.006 & $\bar{~} 0.169$ & 0.298 & 0.108 & $\overline{0.204}$ & 0.095 \\
\hline & & NEW & 0.013 & -0.009 & 0.182 & 0.323 & 0.126 & 0.227 & 0.104 \\
\hline & & $\mathrm{RM}$ & 0.011 & -0.011 & 0.184 & 0.325 & 0.127 & 0.230 & 0.105 \\
\hline & & ICP & 0.013 & -0.014 & 0.182 & 0.322 & 0.129 & 0.226 & 0.104 \\
\hline & \multirow{4}{*}{$40 \%$} & Omni & 0.009 & -0.006 & 0.169 & 0.298 & 0.108 & 0.204 & 0.095 \\
\hline & & NEW & 0.014 & -0.011 & 0.206 & 0.390 & 0.133 & 0.267 & 0.124 \\
\hline & & RM & 0.008 & -0.025 & 0.204 & 0.387 & 0.134 & 0.268 & 0.123 \\
\hline & & ICP & 0.016 & -0.027 & 0.217 & 0.403 & 0.148 & 0.276 & 0.127 \\
\hline
\end{tabular}

Table 1: Simulation results for DGP 1 expressed in terms of bias, RMSE, MAE and MAD averaged over $B=500$ repetitions. Average censoring proportions $p_{c}$ are taken in $\{15 \%, 40 \%\}$ at the median, and sample sizes are taken in $\{100,200\}$.

present substantial biases in the parameter estimation for this DGP, particularly for the intercept. In contrast, NEW exhibits difficulties in terms of bias only for the most complicated situations considered here, that is, for high quantile levels with respect to the censoring proportion. These bias results concerning the estimation of $\beta_{0}$ naturally impact the RMSE and MAE of both RM and ICP. However, while ICP presents considerable biases for both parameters to be estimated, RM quite surprisingly seems to compensate its difficulties for estimating $\beta_{0}$ by an excellent estimation of $\beta_{1}$, especially with respect to Omni. There is little intuition to explain this particular behavior

\begin{tabular}{|c|c|c|c|c|c|c|c|c|c|}
\hline \multirow[b]{2}{*}{$p_{c}$} & \multirow[b]{2}{*}{$\tau$} & \multirow[b]{2}{*}{ Method } & \multicolumn{2}{|c|}{ Bias } & \multicolumn{2}{|c|}{ RMSE } & \multicolumn{2}{|c|}{ MAE } & \multirow[b]{2}{*}{ MAD } \\
\hline & & & $\beta_{0}$ & $\beta_{1}$ & $\beta_{0}$ & $\beta_{1}$ & $\beta_{0}$ & $\beta_{1}$ & \\
\hline \multirow{8}{*}{$30 \%$} & \multirow{5}{*}{0.3} & Omni & 0.021 & 0.014 & 0.373 & 0.507 & 0.244 & 0.337 & 0.466 \\
\hline & & NEW & 0.014 & 0.031 & 0.388 & 0.509 & 0.258 & 0.350 & 0.479 \\
\hline & & $\mathrm{RM}$ & -0.116 & 0.057 & 0.400 & 0.486 & 0.265 & 0.321 & 0.473 \\
\hline & & ICP & -0.071 & 0.140 & 0.400 & 0.582 & 0.264 & 0.363 & 0.513 \\
\hline & & Omni & 0.014 & -0.013 & 0.371 & 0.502 & 0.243 & 0.332 & 0.456 \\
\hline & \multirow{3}{*}{0.7} & NEW & -0.176 & 0.049 & 0.445 & 0.502 & 0.293 & 0.324 & 0.538 \\
\hline & & $\mathrm{RM}$ & -0.403 & 0.104 & 0.560 & 0.456 & 0.404 & 0.311 & 0.581 \\
\hline & & ICP & -0.317 & 0.218 & 0.570 & 0.732 & 0.417 & 0.504 & 0.691 \\
\hline \multirow{8}{*}{$60 \%$} & \multirow{5}{*}{0.3} & Omni & 0.021 & 0.014 & 0.373 & 0.507 & 0.244 & 0.337 & 0.466 \\
\hline & & NEW & -0.058 & 0.075 & 0.390 & 0.503 & 0.268 & 0.338 & 0.473 \\
\hline & & RM & -0.517 & 0.102 & 0.627 & 0.414 & 0.524 & 0.290 & 0.613 \\
\hline & & ICP & -1.097 & 0.442 & 1.208 & 0.792 & 1.083 & 0.502 & 1.204 \\
\hline & & Omni & 0.002 & 0.009 & 0.350 & 0.473 & 0.226 & 0.308 & 0.432 \\
\hline & \multirow{3}{*}{0.5} & NEW & -0.269 & 0.113 & 0.504 & 0.564 & 0.339 & 0.353 & 0.565 \\
\hline & & RM & -0.744 & 0.190 & 0.828 & 0.475 & 0.760 & 0.346 & 0.815 \\
\hline & & ICP & -1.387 & 0.477 & 1.487 & 0.856 & 1.395 & 0.566 & 1.486 \\
\hline
\end{tabular}

Table 2: Simulation results for DGP 2 with sample size $n=200$, expressed in terms of bias, RMSE, MAE and MAD averaged over $B=500$ repetitions. Average censoring proportions $p_{c}$ are taken in $\{30 \%, 60 \%\}$, and quantile levels $\tau$ are chosen among $\{0.3,0.5,0.7\}$. 


\begin{tabular}{|c|c|c|c|c|c|c|c|c|c|}
\hline \multirow[b]{2}{*}{$n$} & \multirow[b]{2}{*}{$p_{c}$} & \multirow[b]{2}{*}{ Method } & \multicolumn{2}{|c|}{ Bias } & \multicolumn{2}{|c|}{ RMSE } & \multicolumn{2}{|c|}{ MAE } & \multirow[b]{2}{*}{ MAD } \\
\hline & & & $\beta_{0}$ & $\beta_{1}$ & $\beta_{0}$ & $\beta_{1}$ & $\beta_{0}$ & $\beta_{1}$ & \\
\hline \multirow{8}{*}{200} & \multirow{4}{*}{$30 \%$} & Omni & "0.007 & -0.016 & $\overline{00.346}$ & 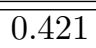 & 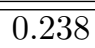 & 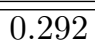 & $\overline{00.403}$ \\
\hline & & NEW & -0.030 & -0.085 & 0.367 & 0.444 & 0.242 & 0.328 & 0.435 \\
\hline & & $\mathrm{RM}$ & -0.202 & -0.201 & 0.392 & 0.463 & 0.284 & 0.334 & 0.467 \\
\hline & & ICP & -0.146 & -0.660 & 0.440 & 0.909 & 0.297 & 0.655 & 0.725 \\
\hline & \multirow{4}{*}{$60 \%$} & Omni & 0.007 & -0.016 & 0.346 & 0.421 & 0.238 & 0.292 & 0.403 \\
\hline & & NEW & -0.302 & -0.340 & 0.490 & 0.659 & 0.369 & 0.509 & 0.640 \\
\hline & & $\mathrm{RM}$ & -0.737 & -0.444 & 0.806 & 0.618 & 0.744 & 0.498 & 0.854 \\
\hline & & ICP & -1.373 & -1.330 & 1.469 & 1.600 & 1.408 & 1.220 & 1.771 \\
\hline \multirow{8}{*}{500} & \multirow{4}{*}{$30 \%$} & $\overline{O m n i}$ & $2-0.001$ & 0.017 & 0.225 & 0.286 & 0.160 & 0.207 & 0.273 \\
\hline & & NEW & -0.021 & -0.043 & 0.235 & 0.316 & 0.165 & 0.228 & 0.297 \\
\hline & & $\mathrm{RM}$ & -0.180 & -0.172 & 0.284 & 0.330 & 0.200 & 0.250 & 0.338 \\
\hline & & ICP & -0.101 & -0.723 & 0.287 & 0.850 & 0.202 & 0.709 & 0.656 \\
\hline & \multirow{4}{*}{$60 \%$} & Omni & -0.001 & 0.017 & 0.225 & 0.286 & 0.160 & 0.207 & 0.273 \\
\hline & & NEW & -0.159 & -0.206 & 0.340 & 0.493 & 0.243 & 0.390 & 0.458 \\
\hline & & RM & -0.638 & -0.360 & 0.676 & 0.472 & 0.644 & 0.398 & 0.705 \\
\hline & & ICP & -1.298 & -1.529 & 1.343 & 1.711 & 1.303 & 1.376 & 1.755 \\
\hline
\end{tabular}

Table 3: Simulation results for DGP 3 for the estimation of the median with sample size $n \in\{200,500\}$ and average censoring proportions $p_{c} \in\{30 \%, 60 \%\}$. Results are expressed in terms of bias, RMSE, MAE and MAD averaged over $B=500$ repetitions.

in this more complicated DGP, although part of the explanation could probably be related to the general arguments concerning the redistribution-of-mass pointed out by Wey et al. (2014) in their Supplementary Materials to which the interested reader is referred. Additionally, similar ambiguous results for the latter technique were observed when considering an alternative estimator for $F_{T \mid \boldsymbol{X}}$, for instance using survival trees as proposed in Wey et al. and using the R code available on their website. In opposition to these results, NEW here satisfactorily mimics the behavior of the omniscient procedure. Simulations for larger samples sizes reveal the same patterns here, and are therefore omitted.

Next, to grasp the impact of a conditional censoring distribution, Table 3 highlights the results of DGP 3 for both sample sizes as well as both censoring percentages for the case $\tau=0.5$. Similar findings to those previously-described still apply here, as the results of NEW still convincingly mimic the benchmark omniscient estimator in comparison to RM and ICP, especially when considering difficult estimation scenarios. These considerations are naturally reflected when it comes to prediction, as NEW confidently outperforms in this setting both considered weighting schemes.

Moving to multivariate covariates, Table 4 reports the results of DGP 4 where four covariates are considered for $\tau=0.5$. For ease of presentation, the results of this DGP are aggregated with respect to the parameters. More specifically, the reported bias stands here for the sum over all parameters of bias taken in absolute value, RMSE stands for the root of mean squared errors summed over all parameters, and MAD stands for the sum over all parameters of median absolute error. Note that in this DGP, and similarly to DGP 1, Portnoy's or Peng and Huang's estimators would a priori be the most appropriate candidates for estimating $\beta_{\tau}$ given their inherent global linear assumption. However, as already mentioned earlier, we are mainly interested here in comparing the proposed procedure with the two weighting techniques that have engendered further research in alternative models. One such model is the multivariate example of variable selection in linear regression for which ICP and RM were adapted in Shows et al. (2010) and Wang et al. (2013), respectively.

As can be observed, the obtained results exhibit analogous patterns to those of the univariate settings; NEW takes advantage of superior bias results to outperform its competitors especially when the proportion of censored responses is important. As a consequence, the predictive potential of NEW also outperforms its competitors in this multivariate setting. Finally, it is worth stressing 


\begin{tabular}{cc||ccc|c||ccc|c}
\multicolumn{1}{c}{$n=200$} & \multicolumn{4}{c}{$n=500$} \\
\multicolumn{1}{c|}{$p_{c}$} & Method & Bias & RMSE & MAE & MAD & Bias & RMSE & MAE & MAD \\
\hline \hline \multirow{3}{*}{$30 \%$} & Omni & 0.018 & 0.213 & 0.320 & 0.177 & 0.014 & 0.135 & 0.202 & 0.112 \\
& NEW & 0.062 & 0.270 & 0.405 & 0.220 & 0.140 & 0.184 & 0.281 & 0.150 \\
& RM & 0.192 & 0.268 & 0.409 & 0.219 & 0.257 & 0.206 & 0.337 & 0.167 \\
& ICP & 0.271 & 0.352 & 0.547 & 0.283 & 0.252 & 0.248 & 0.379 & 0.199 \\
\cline { 2 - 9 } $60 \%$ & Omni & 0.018 & 0.213 & 0.320 & 0.177 & 0.014 & 0.135 & 0.202 & 0.112 \\
& NEW & 0.224 & 0.394 & 0.595 & 0.304 & 0.313 & 0.293 & 0.457 & 0.237 \\
& RM & 0.581 & 0.448 & 0.731 & 0.364 & 0.559 & 0.364 & 0.608 & 0.296 \\
& ICP & 1.044 & 0.716 & 1.153 & 0.560 & 1.017 & 0.621 & 1.096 & 0.493 \\
\hline \hline
\end{tabular}

Table 4: Simulation results for DGP 4 for the estimation of the median. Results are in terms of aggregated over the parameters bias, RMSE, MAE and MAD averaged over $B=500$ repetitions.

out that for this DGP, the estimator of Wey et al. may suppositionally appear more appropriate to embody the technique of redistribution-of-mass than Wang and Wang's estimator, as the former was proposed to avoid kernel smoothing of $F_{T \mid X}$ when confronted to multivariate covariates. However, despite using the available code and tuning the parameters with the recommendations of the paper, the results of Wey et al.'s estimator were here subject to numerical instabilities when studying high censoring proportions, hereby preventing an objective comparison of the methodologies for the considered DGP. Additionally, for lower censoring proportions, the obtained results were analogous to those of RM, and are therefore omitted here in Table 4.

Lastly, to evaluate the effectiveness of the percentile bootstrap described in Section 3 for the proposed procedure, we compare the performance of the latter with the percentile bootstrap of RM for DGPs 1 and 2. Table 5 reports the empirical coverage probability and empirical mean length of the confidence intervals obtained with 300 bootstrapped samples at each of 500 iterations. The nominal level is taken to be 0.95 , and for both procedures the bandwidths are fixed at 0.05 for DGP 1 and 0.10 for DGP 2. As can first be observed, for the simplest DGP 1 both procedures yield coverage probabilities adequately close to the chosen nominal level and with analogous mean empirical length of the confidence intervals. Secondly, for a more complicated scenario with namely more censoring, we observe here that NEW still appropriately approaches the nominal level while RM exhibits difficulties for the latter. We note that this could partly be due to an inappropriate choice of bandwidth. Nevertheless, these results suggest here that the percentile bootstrap represents a satisfactory tool for inference purposes when considering the estimator NEW.

To conclude, from the described simulation results we observe that NEW provides a valuable complement to the literature, especially in cases where the latter seem to fail when confronted to high censoring percentages and quantile levels. Moreover, for the simpler setting considered here, NEW offers similar results to those of RM which are often taken as primary reference. These considerations are believed to be encouraging for the practical use of the newly proposed loss function for quantile regression models.

\section{Real Data Analysis}

As an illustration, we propose to apply the developed methodology to the Channing House dataset which is readily available in the $\mathrm{R}$ package boot. The data consists of 462 records of residents living at the retirement center 'Channing House' during the period January 1964 to July 1975. One of the purposes of the study was to assess the difference between the survival of men and women under the retirement center program, taking age of entry into account. To that end, the dataset contains information about the survival time (in months) of individuals, their sex and their age (in months) at the time they entered the retirement center. At the end of the study, only 176 


\begin{tabular}{|c|c|c|c|c|c|c|c|c|c|c|}
\hline \multirow[b]{3}{*}{ DGP } & \multirow[b]{3}{*}{$p_{c}$} & \multirow[b]{3}{*}{$n$} & \multicolumn{4}{|c|}{$\mathrm{ECP}$} & \multicolumn{4}{|c|}{ EML } \\
\hline & & & \multicolumn{2}{|c|}{$\mathrm{RM}$} & \multicolumn{2}{|c|}{ NEW } & \multicolumn{2}{|c|}{$\mathrm{RM}$} & \multicolumn{2}{|c|}{ NEW } \\
\hline & & & $\beta_{0}$ & $\beta_{1}$ & $\beta_{0}$ & $\beta_{1}$ & $\beta_{0}$ & $\beta_{1}$ & $\beta_{0}$ & $\beta_{1}$ \\
\hline \multirow{4}{*}{$1(\tau=0.5)$} & \multirow{2}{*}{$15 \%$} & 100 & 0.954 & 0.952 & 0.948 & 0.952 & 1.049 & 1.894 & 1.052 & 1.902 \\
\hline & & 200 & 0.936 & 0.950 & 0.938 & 0.960 & 0.752 & 1.342 & 0.752 & 1.346 \\
\hline & \multirow{2}{*}{$40 \%$} & 100 & 0.956 & 0.958 & 0.966 & 0.970 & 1.162 & 2.258 & 1.187 & 2.292 \\
\hline & & 200 & 0.940 & 0.952 & 0.940 & 0.954 & 0.839 & 1.587 & 0.837 & 1.589 \\
\hline \multirow{4}{*}{$2(\tau=0.3)$} & \multirow{2}{*}{$30 \%$} & 100 & 0.956 & 0.956 & 0.960 & 0.948 & 2.085 & 2.692 & 2.218 & 2.835 \\
\hline & & 200 & 0.922 & 0.946 & 0.972 & 0.954 & 1.490 & 1.872 & 1.543 & 1.990 \\
\hline & \multirow{2}{*}{$60 \%$} & 100 & 0.758 & 0.944 & 0.968 & 0.942 & 1.864 & 2.315 & 2.301 & 2.892 \\
\hline & & 200 & 0.756 & 0.950 & 0.960 & 0.966 & 1.463 & 1.716 & 1.609 & 1.970 \\
\hline
\end{tabular}

Table 5: Bootstrap results for DGP 1 and 2 based on 500 simulations with 300 bootstrap samples. ECP and EML stand respectively for empirical coverage probability and empirical mean length, for a nominal level of 0.95 . Average censoring proportions $p_{c}$ are taken in $\{15 \%, 30 \%, 40 \%, 60 \%\}$, and quantile levels $\tau$ are chosen among $\{0.3,0.5\}$.

residents taken into account experienced the event of interest, resulting in approximately $62 \%$ of censoring. Further information on the dataset may be found for instance in Hyde (1980).

Embracing the role of a practitioner, as a preliminary remark in the objective of fitting a quantile regression model to the data, we note that the responses are subject to a considerable amount of censoring, hereby ruling out a confident use of inverse-censoring-probability-based methodologies, and in particular Bang and Tsiatis' estimator as illustrated in our simulations. Therefore, we will only consider for this analysis Wang and Wang's estimator as a point of comparison for the proposed methodology.

In order to examine the effects of the covariates on several quantile levels of the survival time, we now consider first a sequence of quantiles equally ranging from 0.1 to 0.5 with 0.05 increments. For every quantile level, we estimate $\beta_{\tau}$ with both Wang and Wang's estimator and the proposed methodology. Implementation of the latter is carried out using the algorithm of Section 4, where, as in Section 5, we adopt Beran's estimator on each sex for $\widehat{G}_{C}$. Wang and Wang's estimator is likewise implemented with Beran's estimator on each sex for $\widehat{F}_{T \mid X}$. Concerning the bandwidths of both estimators, equivalently to our simulation study, the latter are chosen using 5 -fold cross validation on the normalized covariate age among 15 candidates equally ranging from 0.05 to 1.5. Finally, for both procedures, $95 \%$ confidence intervals are constructed using the percentile bootstrap described in Section 3 based on 300 bootstrap samples. For computational convenience, all bootstrap estimations for both methodologies are based on the same bandwidths as for the initial dataset.

Estimation results are presented in Figure 2. A first consideration one may acknowledge from the presented figure is the noticeable numerical stability offered by the proposed methodology in comparison to Wang and Wang's estimator when estimating relatively high quantile levels with respect to the censoring proportion. This appears as a natural consequence of requiring for our procedure the estimation of $G_{C}$ rather than $F_{T \mid X}$, hereby highlighting again the potential complement our estimator may provide to the literature for relatively highly censored datasets. Apart from this first examination, considering only quantile levels below the region of numerical instability of RM, both methods suggest unsurprisingly a significant negative effect of age at entry on the survival time, while there seems to be a discrepancy on the significance of a gender effect for the present dataset even though the upper confidence band of RM is very close to fully incorporating the value 0 . Lastly, examining the bootstrap confidence intervals, we observe that confidence intervals for NEW tend to be a little smaller than for RM except for the covariate sex. Furthermore, it is worth stressing out that the width of the NEW's confidence intervals does not seem here to fluctuate much with the increasing quantile levels of interest, hereby exposing a confident resistance to the risk of estimating higher quantiles with a consequent proportion of 

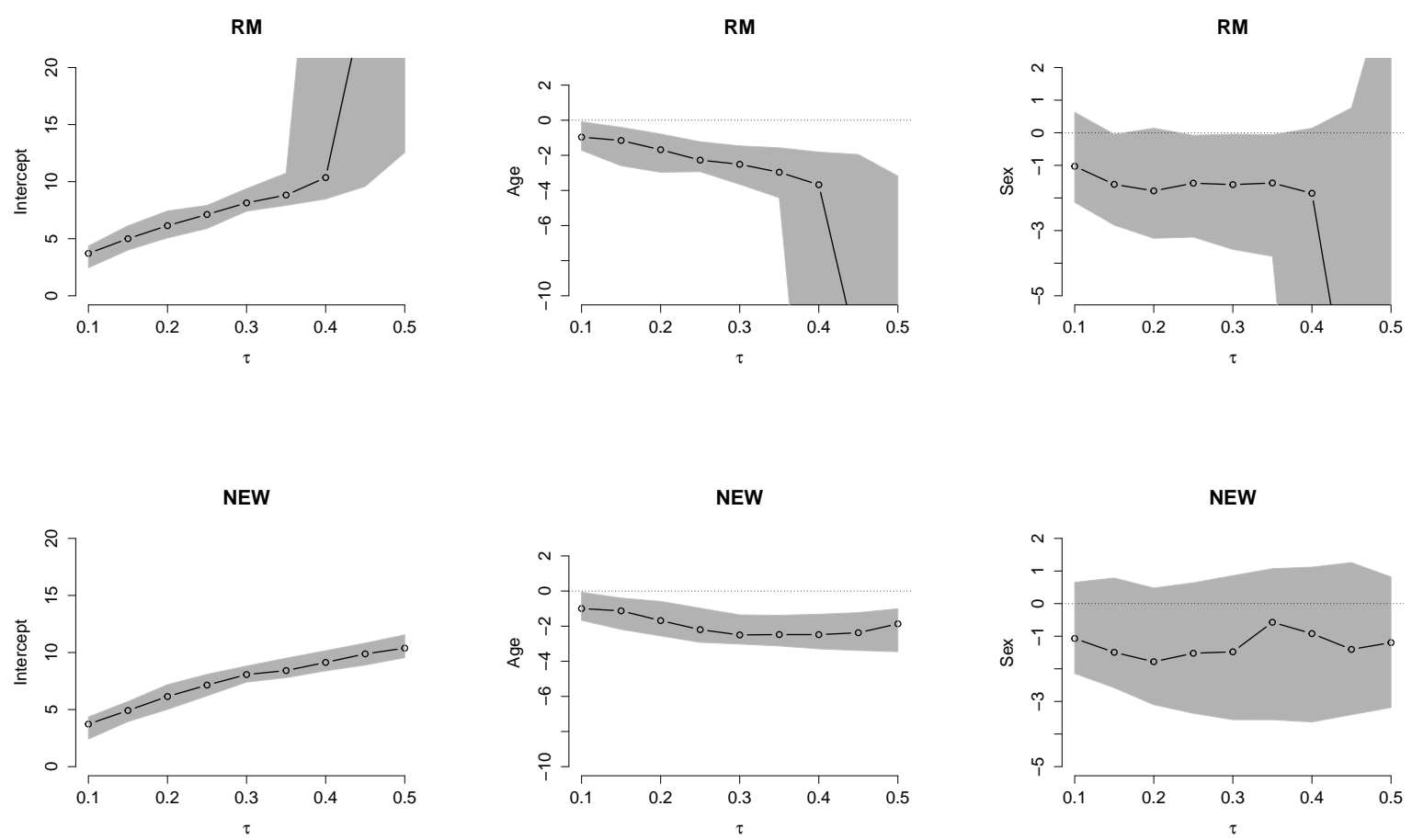

Figure 2: Channing House data. Estimated quantile coefficients for regressing the survival time (in years) on sex and age at entry (standardized). The top row reports the results for Wang and Wang's estimator (RM), while the bottom row depicts results obtained with the present methodology (NEW). Shaded areas correspond to $95 \%$ confidence intervals based on 300 bootstrap samples.

censored responses.

To further examine the performance of both procedures for the present dataset, we consider a prediction study based on cross validation. Specifically, we first randomly split the data into a training set of 350 observations and a testing set of 112 observations. For quantile levels ranging only from 0.1 to 0.4 by 0.05 increments given the instability of $\mathrm{RM}$ beyond $\tau=0.4$, we then estimate the quantile coefficients for each method based on the training set, and consequently evaluate the predictive performance of the $\tau$-th conditional quantile of fully observed responses in the testing set. As an evaluation measure, we therefore consider the following prediction error:

$$
\mathrm{PE}=\operatorname{med}_{\substack{i \in \text { testing } \\ \Delta_{i}=1}} \rho_{\tau}\left(Y_{i}-\hat{\beta}_{\tau}^{\top} \boldsymbol{X}_{i}\right)
$$

where $\widehat{\beta}_{\tau}$ is an estimator of $\beta_{\tau}$ based on the training set, and $Y_{i}, i=1, \ldots, n$, denote the observed survival times. For robust results, this cross validation is repeated 200 times and the median of all PE's is reported in Table 6. Similarly to the above-described bootstrap confidence intervals, for computational consideration, the bandwidths of both NEW and RM are selected by cross validation on one initial training set. Results suggest that for this dataset, the prediction accuracies of both procedures are very similar, with a slight overall advantage for NEW. Given the further improvement of NEW over RM for quantile levels lying above $\tau=0.4$, this observation may then advocate here for the practical choice of the proposed methodology for a robust quantile regression analysis. 


\begin{tabular}{c||ccccccc} 
Method & $\tau=0.1$ & $\tau=0.15$ & $\tau=0.2$ & $\tau=0.25$ & $\tau=0.3$ & $\tau=0.35$ & $\tau=0.4$ \\
\hline \hline \multirow{2}{*}{ RM } & 0.474 & 0.647 & 0.876 & 1.112 & 1.225 & 1.522 & 2.103 \\
& $(0.02)$ & $(0.04)$ & $(0.07)$ & $(0.10)$ & $(0.10)$ & $(0.11)$ & $(0.10)$ \\
NEW & 0.474 & 0.650 & 0.853 & 1.065 & 1.225 & 1.437 & 1.774 \\
& $(0.02)$ & $(0.03)$ & $(0.05)$ & $(0.05)$ & $(0.11)$ & $(0.11)$ & $(0.10)$ \\
\hline \hline
\end{tabular}

Table 6: Channing House data. Median prediction error over 200 cross validations, with standard deviations of the latter in parentheses, for quantile levels $\tau \in\{0.1, \ldots, 0.4\}$ for both RM and NEW.

\section{Conclusion}

In this work we have proposed an adapted version of the check function for evaluating sample quantiles or more generally quantile regression when confronted to possible right-censored responses. By tackling the problem of incomplete data at the level of the loss function through an adjustment of the underlying penalization for under- and overestimation of the true quantile value, the proposed loss function allows to plainly take profit of all observations at hand. This has to be contrasted for instance to the prominent inverse-censoring-probability weighting scheme of Koul et al. (1981).

As an illustration of the newly proposed loss function for regression models, we proposed to consider the particular and well-studied case of a simple linear regression, for which consistency and asymptotic normality of the quantile coefficient estimator were obtained using recent results on non-smooth semiparametric estimation equations with an infinite-dimensional nuisance parameter. For practical implementation, a detailed adaptation of the MM algorithm was then proposed, and an extensive simulation study was carried out to illustrate the finite sample performance of the methodology. From the latter study, the proposed estimator was observed to perform competitively with respect to the established technique of redistribution-of-mass, especially when considering highly censored datasets. This consideration was highlighted in a real data application as well. Furthermore, for simpler settings, the proposed estimator revealed to be interestingly robust to both the sample size and the smoothing parameter to be selected for a preliminary, and possibly local, estimator of the censoring distribution on which the procedure is built. Lastly, for multivariate settings, while the present paper illustrates the use of Beran's estimator for the conditional censoring distribution, we note that alternative modelling techniques for the latter may be more appropriate to avoid the curse of dimensionality, such as a Cox model, a single-index model or survival trees for instance. Such developments deserve further analysis.

To conclude, considering now a broader picture than strictly parametric regression, the proposed results also illustrate the potential of the studied loss function for improvement of various modelling techniques that are currently built upon the inverse-censoring-probability weights, such as single-index regression or copula-based regression to name a few. This provides encouraging perspectives for enhancement of flexible censored quantile regression models.

\section{Acknowledgments}

All authors acknowledge financial support from IAP research network P7/06 of the Belgian Government (Belgian Science Policy). M. De Backer and A. El Ghouch further acknowledge financial support from the FSR project IMAQFSR15PROJEL from the Fonds de la Recherche Scientifique de Belgique (F.R.S.-FNRS). I. Van Keilegom also acknowledges support from the European Research Council (2016-2021, Horizon 2020 / ERC grant agreement No. 694409).

Computational resources have been provided by the supercomputing facilities of the Université catholique de Louvain (CISM/UCL) and the Consortium des Équipements de Calcul Intensif en Fédération Wallonie Bruxelles (CÉCI) funded by the Fonds de la Recherche Scientifique de Belgique under convention 2.5020.11. 


\section{Appendix}

We provide in this appendix the proofs of Section 3. Consistency of the proposed estimator relies heavily on the work of Delsol and Van Keilegom (2015) (DVK hereafter) on non-smooth semiparametric $M$-estimation problems, while the proof of asymptotic normality relies on a modified version of Theorem 2 of Chen et al. (2003) proposed in Birke et al. (2017). As a preliminary remark, and as already mentioned in Section 3, note that the following proofs are built on a crucial result of Lopez (2011) for the class $\mathcal{G}$ in (C3). This implies that our proofs have to be read as such under similar conditions as for Lopez, that is, assuming the existence of a function $g: \mathbb{R}^{d+1} \rightarrow \mathbb{R}$ such that $G_{C}(\cdot \mid \boldsymbol{X})=G_{C}(\cdot \mid g(\boldsymbol{X}))$, or simply considering the case of a univariate covariate. For ease of reading, the proofs are written considering the latter case.

Proof of Theorem 3.1. In Theorem 1 of DVK, five high level conditions (A1)-(A5) are developed under which an $M$-estimator is consistent in a general semiparametric maximization problem. We therefore only need to verify the latter conditions to prove the consistency of $\widehat{\beta}_{\tau}$. For notational convenience with respect to the work of DVK, let us first rewrite $\widehat{\beta}_{\tau}$ as

$$
\widehat{\beta}_{\tau}=\arg \max _{\beta \in \mathcal{B}} \sum_{i=1}^{n} \psi_{\tau}\left(\beta^{\top} \boldsymbol{X}_{i} ; Y_{i}, \widehat{G}_{C}\left(\cdot \mid \boldsymbol{X}_{i}\right)\right)
$$

where $\psi_{\tau}(a ; y, G)=-\rho_{\tau}(a ; y)+(1-\tau) \int_{0}^{a} G(s) \mathrm{d} s$ and where $\mathcal{B}$ is a compact parameter space, taken to be the neighborhood of $\beta_{\tau}$ mentioned in our assumptions. For further convenience and without any loss of generality, the proof is written considering the response variable $Y$ to be positive. This consideration is solely done in the purpose of being coherent with the arbitrary set value of 0 in the correcting term of $\psi_{\tau}$ with respect to $v$ defined in assumption (C3). For instance, one could also easily consider in the following a strictly negative variable $Y$, but this would possibly require the arbitrary chosen constant 0 to be replaced by any constant below $v$ as we only wish to control the behavior of the nuisance parameter $\widehat{G}_{C}(\cdot \mid \boldsymbol{X})$ below this $v$.

Next, starting with condition (A1) in DVK, note that the latter is readily satisfied in our framework by construction of $\widehat{\beta}_{\tau}$. Furthermore, using the definition of $\mathcal{G}$ in (C3) as the space embedding the nuisance parameter $G_{C}$, and equiping the latter with the distance $d_{\mathcal{G}}\left(G_{1}, G_{2}\right)=$ $\sup _{\boldsymbol{x} \in \operatorname{supp}(\boldsymbol{X})} \sup _{y \leq v}\left|G_{1}(y \mid \boldsymbol{x})-G_{2}(y \mid \boldsymbol{x})\right|$ for any $G_{1}, G_{2} \in \mathcal{G}$, note that (A3) in DVK is straightforwardly satisfied as well provided assumption (C5)-(i) holds. We therefore only need to verify here conditions (A2), (A4) and (A5).

Starting with the identifiability condition (A2) ensuring the uniqueness of $\beta_{\tau}$, we need to verify that for any $\epsilon>0, \inf _{\left\|\beta-\beta_{\tau}\right\|>\epsilon} \mathbb{E}\left[\psi_{\tau}\left(\beta_{\tau}^{\top} \boldsymbol{X} ; Y, G_{C}(\cdot \mid \boldsymbol{X})\right)-\psi_{\tau}\left(\beta^{\top} \boldsymbol{X} ; Y, G_{C}(\cdot \mid \boldsymbol{X})\right)\right]>0$, where $\|\cdot\|$ denotes the Euclidean distance. To that end, using the definition of $\varphi_{\tau}$, we can show that

$$
\begin{aligned}
& \inf _{\left\|\beta-\beta_{\tau}\right\|>\epsilon} \mathbb{E}\left[\psi_{\tau}\left(\beta_{\tau}^{\top} \boldsymbol{X} ; Y, G_{C}(\cdot \mid \boldsymbol{X})\right)-\psi_{\tau}\left(\beta^{\top} \boldsymbol{X} ; Y, G_{C}(\cdot \mid \boldsymbol{X})\right)\right] \\
= & \inf _{\left\|\beta-\beta_{\tau}\right\|>\epsilon} \mathbb{E}\left[\int_{\beta^{\top} \boldsymbol{X}}^{\beta_{\tau}^{\top} \boldsymbol{X}}\left(\mathbb{1}(Y \geq s)-(1-\tau) \bar{G}_{C}(s \mid \boldsymbol{X})\right) \mathrm{d} s\right] \\
= & \inf _{\left\|\beta-\beta_{\tau}\right\|>\epsilon} \mathbb{E}_{\boldsymbol{X}}\left[\int_{\beta^{\top} \boldsymbol{X}}^{\beta_{\tau}^{\top} \boldsymbol{X}}\left(1-G_{C}(s \mid \boldsymbol{X})\right)\left(\tau-F_{T \mid \boldsymbol{X}}(s \mid \boldsymbol{X})\right) \mathrm{d} s\right] .
\end{aligned}
$$

Under assumptions (C1), (C2) and (C4), the latter expectation is observed to be strictly positive, hereby ensuring condition (A2) is satisfied.

Next, for (A4) to hold, it suffices by Remark 1(ii) in DVK and assumption (C3) to show that the class

$$
\mathcal{F}=\left\{(y, \boldsymbol{x}) \mapsto \psi_{\tau}\left(\beta^{\top} \boldsymbol{x} ; y, G(\cdot \mid \boldsymbol{x})\right): \beta \in \mathcal{B}, G \in \mathcal{G}\right\}
$$

is Glivenko-Cantelli. For this, by Theorem 2.4.1 in Van der Vaart and Wellner (1996), we need to prove that for all $\epsilon>0$, the $\epsilon$-bracketing number $N_{[]}\left(\epsilon, \mathcal{F}, L_{1}(P)\right)$ of the class $\mathcal{F}$ with respect to 
the $L_{1}$ probability measure on $(Y, \boldsymbol{X})$ is finite. To that end, let $\psi_{\tau}=\psi_{\tau 1}+\psi_{\tau 2}+\psi_{\tau 3}$, where

$$
\begin{aligned}
& \psi_{\tau 1}\left(\beta^{\top} \boldsymbol{x} ; y, G(\cdot \mid \boldsymbol{x})\right)=-\tau\left(y-\beta^{\boldsymbol{\top}} \boldsymbol{x}\right) \\
& \psi_{\tau 2}\left(\beta^{\boldsymbol{\top}} \boldsymbol{x} ; y, G(\cdot \mid \boldsymbol{x})\right)=\left(y-\beta^{\boldsymbol{\top}} \boldsymbol{x}\right) \mathbb{1}\left(y<\beta^{\top} \boldsymbol{x}\right) \\
& \psi_{\tau 3}\left(\beta^{\boldsymbol{\top}} \boldsymbol{x} ; y, G(\cdot \mid \boldsymbol{x})\right)=(1-\tau) \int_{0}^{\beta^{\top} \boldsymbol{x}} G(s \mid \boldsymbol{x}) \mathrm{d} s,
\end{aligned}
$$

and let $\mathcal{F}_{1}, \mathcal{F}_{2}$ and $\mathcal{F}_{3}$ denote the classes induced by $\psi_{\tau 1}, \psi_{\tau 2}$ and $\psi_{\tau 3}$, respectively. From this decomposition, it is easy to see that

$$
N_{[]}\left(\epsilon, \mathcal{F}, L_{1}(P)\right) \leq \prod_{j=1}^{3} N_{[]}\left(\epsilon, \mathcal{F}_{j}, L_{1}(P)\right) .
$$

Now, for the classes $\mathcal{F}_{1}$ and $\mathcal{F}_{2}$, suppose for simplicity and without loss of generality that all coordinates of $\boldsymbol{x}$ are positive, and define $M_{\epsilon}=O\left(\epsilon^{-2}\right)$ pairs $\left(\beta_{k}^{L}, \beta_{k}^{U}\right), k=1, \ldots, M_{\epsilon}$, that cover $\mathcal{B}$, assumed to be compact by $(\mathrm{C} 1)$, such that $\left(\beta_{k}^{L_{\top}} \boldsymbol{x}, \beta_{k}^{U_{\top}} \boldsymbol{x}\right)$ define brackets of length $\epsilon \tau /(1-\tau)$ for the class $\left\{\boldsymbol{x} \mapsto \beta^{\top} \boldsymbol{x}: \beta \in \mathcal{B}\right\}$ with respect to the $L_{1}$-norm. Then, it is straightforward that $N_{[]}\left(\epsilon, \mathcal{F}_{j}, L_{1}(P)\right) \leq K_{j} \epsilon^{-2}$ for some finite constants $K_{j}>0, j=1,2$, which, combined with (A.2), suggests we only have to verify that $N_{[]}\left(\epsilon, \mathcal{F}_{3}, L_{1}(P)\right)$ is bounded in order to prove that condition (A4) holds in our framework.

To that end, by Lemma 6.1 in Lopez (2011) which extends Theorem 2.7.5 in Van der Vaart and Wellner, first note that there exist $N_{\epsilon} \leq \exp \left(K_{3} \epsilon^{-2 /(1+\eta)}\right)$ brackets $\left(\underline{G}_{j}, \bar{G}_{j}\right), j=1, \ldots, N_{\epsilon}$, for a finite constant $K_{3}>0$ such that, under (C3), for all $G \in \mathcal{G}$, there exists $j=1, \ldots, N_{\epsilon}$, for which $\underline{G}_{j} \leq G \leq \bar{G}_{j}$, and

$$
\int_{\operatorname{supp}(\boldsymbol{X})} \int_{0}^{v}\left|\bar{G}_{j}(s \mid \boldsymbol{x})-\underline{G}_{j}(s \mid \boldsymbol{x})\right| \mathrm{d} s \mathrm{~d} F_{\boldsymbol{X}}(\boldsymbol{x})<\epsilon,
$$

where $F_{\boldsymbol{X}}(\boldsymbol{x})$ denotes the c.d.f. of $\boldsymbol{X}$. From this result, our claim for (A4) to hold is that brackets for $\mathcal{F}_{3}$ are given by $\left(\underline{\zeta}_{j k}, \bar{\zeta}_{j k}\right), j=1, \ldots, N_{\epsilon}, k=1, \ldots, M_{\epsilon}$, where

$$
\begin{aligned}
& \underline{\zeta}_{j k}(\boldsymbol{x})=(1-\tau) \int_{0}^{\beta_{k}^{L_{\top}} \boldsymbol{x}} \underline{G}_{j}(s \mid \boldsymbol{x}) \mathrm{d} s, \\
& \bar{\zeta}_{j k}(\boldsymbol{x})=(1-\tau) \int_{0}^{\beta_{k}^{U_{\top}} \boldsymbol{x}} \bar{G}_{j}(s \mid \boldsymbol{x}) \mathrm{d} s .
\end{aligned}
$$

For this claim to hold, as it is straightforward to verify that for all $\zeta \in \mathcal{F}_{3}$ there exist $j=1, \ldots, N_{\epsilon}$, and $k=1, \ldots, M_{\epsilon}$, such that $\underline{\zeta}_{j k} \leq \zeta \leq \bar{\zeta}_{j k}$, we only need to show that

$$
\int_{\operatorname{supp}(\boldsymbol{X})}\left|\bar{\zeta}_{j k}(\boldsymbol{x})-\underline{\zeta}_{j k}(\boldsymbol{x})\right| \mathrm{d} F_{\boldsymbol{X}}(\boldsymbol{x})<\epsilon, \quad j=1, \ldots, N_{\epsilon}, k=1, \ldots, M_{\epsilon}
$$

To that end, developing the expressions of $\underline{\zeta}_{j k}$ and $\bar{\zeta}_{j k}$, we have that

$$
\begin{aligned}
& \int_{\operatorname{supp}(\boldsymbol{X})}\left|\bar{\zeta}_{j k}(\boldsymbol{x})-\underline{\zeta}_{j k}(\boldsymbol{x})\right| \mathrm{d} F_{\boldsymbol{X}}(\boldsymbol{x}) \\
= & (1-\tau) \int_{\operatorname{supp}(\boldsymbol{X})}\left|\int_{0}^{\beta_{k}^{U_{\top}} \boldsymbol{x}} \bar{G}_{j}(s \mid \boldsymbol{x}) \mathrm{d} s-\int_{0}^{\beta_{k}^{L_{\top}} \boldsymbol{x}} \underline{G}_{j}(s \mid \boldsymbol{x}) \mathrm{d} s\right| \mathrm{d} F_{\boldsymbol{X}}(\boldsymbol{x}),
\end{aligned}
$$

where the latter expression can be bounded above by $T_{1}+T_{2}$ where

$$
\begin{aligned}
& T_{1}=(1-\tau) \int_{\operatorname{supp}(\boldsymbol{X})}\left|\int_{0}^{\beta_{k}^{U \top} \boldsymbol{x}} \bar{G}_{j}(s \mid \boldsymbol{x}) \mathrm{d} s-\int_{0}^{\beta_{k}^{U_{\top}} \boldsymbol{x}} \underline{G}_{j}(s \mid \boldsymbol{x}) \mathrm{d} s\right| \mathrm{d} F_{\boldsymbol{X}}(\boldsymbol{x}), \\
& T_{2}=(1-\tau) \int_{\operatorname{supp}(\boldsymbol{X})}\left|\int_{0}^{\beta_{k}^{U \top} \boldsymbol{x}} \underline{G}_{j}(s \mid \boldsymbol{x}) \mathrm{d} s-\int_{0}^{\beta_{k}^{L_{\top}} \boldsymbol{x}} \underline{G}_{j}(s \mid \boldsymbol{x}) \mathrm{d} s\right| \mathrm{d} F_{\boldsymbol{X}}(\boldsymbol{x}) .
\end{aligned}
$$


We will now show that both $T_{1}$ and $T_{2}$ can be bounded above such that their sum is bounded by $\epsilon$. Starting with $T_{1}$, we have that

$$
\begin{aligned}
T_{1} & \leq(1-\tau) \int_{\operatorname{supp}(\boldsymbol{X})} \int_{0}^{\beta_{k}^{U_{\top}} \boldsymbol{x}}\left|\bar{G}_{j}(s \mid \boldsymbol{x})-\underline{G}_{j}(s \mid \boldsymbol{x})\right| \mathrm{d} s \mathrm{~d} F_{\boldsymbol{X}}(\boldsymbol{x}) \\
& \leq(1-\tau) \int_{\operatorname{supp}(\boldsymbol{X})} \int_{0}^{v}\left|\bar{G}_{j}(s \mid \boldsymbol{x})-\underline{G}_{j}(s \mid \boldsymbol{x})\right| \mathrm{d} s \mathrm{~d} F_{\boldsymbol{X}}(\boldsymbol{x}) \leq(1-\tau) \epsilon,
\end{aligned}
$$

using assumption (C4) and (A.3) for the second and last inequalities, respectively. Concentrating now on $T_{2}$, we have that

$$
\begin{aligned}
T_{2} & \leq(1-\tau) \int_{\operatorname{supp}(\boldsymbol{X})} \int_{\beta_{k}^{L_{\top}} \boldsymbol{L _ { T }}}^{\beta_{\top}^{U_{\top}} \boldsymbol{x}}\left|\underline{G}_{j}(s \mid \boldsymbol{x})\right| \mathrm{d} s \mathrm{~d} F_{\boldsymbol{X}}(\boldsymbol{x}) \\
& \leq(1-\tau) \int_{\operatorname{supp}(\boldsymbol{X})}\left|\beta_{k}^{U_{\top}} \boldsymbol{x}-\beta_{k}^{L_{\top}} \boldsymbol{x}\right| \mathrm{d} F_{\boldsymbol{X}}(\boldsymbol{x}) \leq \tau \epsilon,
\end{aligned}
$$

given the brackets induced by $\left(\beta_{k}^{L_{\top}}, \beta_{k}^{U_{\top}}\right)$ for the class $\left\{\boldsymbol{x} \mapsto \beta^{\top} \boldsymbol{x}: \beta \in \mathcal{B}\right\}$ with respect to the $L_{1}$-norm. This completes the proof that $N_{[]}\left(\epsilon, \mathcal{F}_{3}, L_{1}(P)\right)$ is bounded. Hence, we conclude that $N_{[]}\left(\epsilon, \mathcal{F}, L_{1}(P)\right)=O\left(\exp \left(K_{3} \epsilon^{-2 /(1+\eta)}\right)\right)$, from which it follows that condition (A4) holds.

Lastly, for condition (A5), we need to establish that

$$
\lim _{d_{\mathcal{G}}\left(G, G_{C}\right) \rightarrow 0} \sup _{\beta \in \mathcal{B}}\left|\mathbb{E}\left[\psi_{\tau}\left(\beta^{\top} \boldsymbol{X} ; Y, G(\cdot \mid \boldsymbol{X})\right)-\psi_{\tau}\left(\beta^{\top} \boldsymbol{X} ; Y, G_{C}(\cdot \mid \boldsymbol{X})\right)\right]\right|=0
$$

To that end, note that

$$
\begin{aligned}
& \sup _{\beta \in \mathcal{B}}\left|\mathbb{E}\left[\psi_{\tau}\left(\beta^{\top} \boldsymbol{X} ; Y, G(\cdot \mid \boldsymbol{X})\right)-\psi_{\tau}\left(\beta^{\top} \boldsymbol{X} ; Y, G_{C}(\cdot \mid \boldsymbol{X})\right)\right]\right| \\
\leq & (1-\tau) \sup _{\beta \in \mathcal{B}} \mathbb{E}_{\boldsymbol{X}}\left[\int_{0}^{\beta^{\top} \boldsymbol{X}}\left|G(s \mid \boldsymbol{X})-G_{C}(s \mid \boldsymbol{X})\right| \mathrm{d} s\right] .
\end{aligned}
$$

Under assumption $(\mathrm{C} 4)$, this expression can then in turn be bounded above by

$$
(1-\tau) \int_{0}^{v} \sup _{\boldsymbol{x} \in \operatorname{supp}(\boldsymbol{X})}\left|G(s \mid \boldsymbol{x})-G_{C}(s \mid \boldsymbol{x})\right| \mathrm{d} s \leq(1-\tau) v \sup _{\boldsymbol{x} \in \operatorname{supp}(\boldsymbol{X})} \sup _{y \leq v}\left|G(y \mid \boldsymbol{x})-G_{C}(y \mid \boldsymbol{x})\right|,
$$

which converges to 0 when $d_{\mathcal{G}}\left(G, G_{C}\right) \rightarrow 0$, provided assumption (C3) holds. This completes the proof that (A5) holds in our framework. Hence the assumptions of Theorem 1 in DVK are met, from which the weak consistency of $\widehat{\beta}_{\tau}$ follows.

Before developing the proof of Theorem 3.2, we now illustrate in the following Lemma how one could replace the general condition (C5)-(ii) in our assumptions by appropriate bandwidth and kernel conditions when considering the particular case of Beran's estimator described in (2.8).

Lemma 1. Suppose conditions (C2) and (C3) hold, and that the following assumptions for Beran's estimator in (2.8) hold as well:

(C6) The bandwidth $h_{n}$ satisfies $h_{n}=O\left(n^{-\nu}\right)$, for $1 / 4<\nu<1 / 3$.

(C7) The kernel function $K(\cdot) \geq 0$ is compactly supported and Lipschitz continuous of order 1. Furthermore, $\int K(u) \mathrm{d} u=1, \int u K(u) \mathrm{d} u=0$ and $\int K^{2}(u) \mathrm{d} u<\infty$.

Then, uniformly in $\beta \in \mathcal{B}$,

$$
\mathbb{E}_{\boldsymbol{X}}\left[\boldsymbol{X}\left(\widehat{G}_{C}\left(\beta^{\top} \boldsymbol{X} \mid \boldsymbol{X}\right)-G_{C}\left(\beta^{\top} \boldsymbol{X} \mid \boldsymbol{X}\right)\right)\right]=n^{-1} \sum_{i=1}^{n} \boldsymbol{X}_{i} \xi\left(Y_{i}, \Delta_{i}, \beta^{\top} \boldsymbol{X}_{i} \mid \boldsymbol{X}_{i}\right)+o_{\mathbb{P}}\left(n^{-1 / 2}\right),
$$


where $\xi$ takes the following form for a response variable still considered to be positive:

$$
\xi\left(Y_{i}, \Delta_{i}, t \mid \boldsymbol{x}\right)=\left(1-G_{C}(t \mid \boldsymbol{x})\right)\left[\int_{0}^{Y_{i} \wedge t} \frac{-\mathrm{d} H_{0}(s \mid \boldsymbol{x})}{\{1-H(s \mid \boldsymbol{x})\}^{2}}+\frac{\left(1-\Delta_{i}\right) \mathbb{1}\left(Y_{i} \leq t\right)}{\left\{1-H\left(Y_{i} \mid \boldsymbol{x}\right)\right\}}\right],
$$

where $H(t \mid \boldsymbol{x})=\mathbb{P}(Y \leq t \mid \boldsymbol{X}=\boldsymbol{x})$ and $H_{0}(t \mid \boldsymbol{x})=\mathbb{P}(Y \leq t, \Delta=0 \mid \boldsymbol{X}=\boldsymbol{x})$.

Proof of Lemma 1. Using the i.i.d. expansion of $\widehat{G}_{C}(c \mid \boldsymbol{x})$ uniformly in $c$ and $\boldsymbol{x}$, under conditions (C2), (C3), (C6) and (C7), we have (see e.g. Gonzalez-Manteiga and Cadarso-Suarez (1994) or Van Keilegom and Veraverbeke (1997)):

$$
\begin{aligned}
\mathbb{E}_{\boldsymbol{X}}\left[\boldsymbol{X}\left(\widehat{G}_{C}\left(\beta^{\top} \boldsymbol{X} \mid \boldsymbol{X}\right)-G_{C}\left(\beta^{\top} \boldsymbol{X} \mid \boldsymbol{X}\right)\right)\right] \\
=\left(n h_{n}\right)^{-1} \mathbb{E}_{\boldsymbol{X}}\left[\boldsymbol{X} \sum_{i=1}^{n} \frac{K\left(\frac{\boldsymbol{X}-\boldsymbol{X}_{i}}{h_{n}}\right)}{\left(n h_{n}\right)^{-1} \sum_{j=1}^{n} K\left(\frac{\boldsymbol{X}_{-} \boldsymbol{X}_{j}}{h_{n}}\right)} \xi\left(Y_{i}, \Delta_{i}, \beta^{\top} \boldsymbol{X} \mid \boldsymbol{X}\right)\right]+o_{\mathbb{P}}\left(n^{-1 / 2}\right) \\
=\left(n h_{n}\right)^{-1} \sum_{i=1}^{n} \int_{-\infty}^{+\infty} \boldsymbol{x} K\left(\frac{\boldsymbol{x}-\boldsymbol{X}_{i}}{h_{n}}\right) \xi\left(Y_{i}, \Delta_{i}, \beta^{\top} \boldsymbol{x} \mid \boldsymbol{x}\right) \mathrm{d} \boldsymbol{x}+o_{\mathbb{P}}\left(n^{-1 / 2}\right),
\end{aligned}
$$

where $\xi$ is described in (A.4). Observing that the function $\xi\left(Y_{i}, \Delta_{i}, t \mid \boldsymbol{x}\right)$ involves an indicator function with respect to $t$, standard change of variables and a modulus of continuity argument of the empirical distribution function (see Theorem 2.14 in Stute (1982)) combined with assumption (C7) then lead to the desired result.

Proof of Theorem 3.2. Given that $\widehat{\beta}_{\tau}$ is shown to be weakly consistent in Theorem 3.1 and that $\widehat{G}_{C}$ is assumed by (C5)-(i) to be a uniformly consistent estimator of $G_{C}$, to prove that our proposed estimator is asymptotically normally distributed we now restrict the spaces $\mathcal{B}$ and $\mathcal{G}$ to shrinking neighborhoods around the true $\beta_{\tau}$ and $G_{C}$ in order to avoid possible local minima. That is, we define the spaces $\mathcal{B}_{\delta}=\left\{\beta \in \mathcal{B}:\left\|\beta-\beta_{\tau}\right\| \leq \delta_{n}\right\}$ and $\mathcal{G}_{\delta}=\left\{G \in \mathcal{G}: d_{\mathcal{G}}\left(G, G_{C}\right) \leq \delta_{n}\right\}$ for some $\delta_{n}=o(1)$. In this context, we rely for our proof on the work of Birke et al. (2017) which slightly adapts Theorem 2 of Chen et al. (2003).

We therefore need to verify conditions (C.1)-(C.6) of Proposition 2 in Birke et al. (2017) in order to establish the asymptotic normality of our proposed estimator. To that end, let us first define $M_{n}(\beta, G)=n^{-1} \sum_{i=1}^{n} m\left(Y_{i}, \boldsymbol{X}_{i}, \beta, G\right)$, where

$$
m\left(Y_{i}, \boldsymbol{X}_{i}, \beta, G\right)=\boldsymbol{X}_{i}\left((1-\tau)\left(1-G\left(\beta^{\top} \boldsymbol{X}_{i} \mid \boldsymbol{X}_{i}\right)\right)-\mathbb{1}\left(Y_{i}>\beta^{\top} \boldsymbol{X}_{i}\right)\right) .
$$

Furthermore, let

$$
\begin{aligned}
M(\beta, G) & =\mathbb{E}[m(Y, \boldsymbol{X}, \beta, G)] \\
& =\mathbb{E}_{\boldsymbol{X}}\left[\boldsymbol{X}\left\{(1-\tau)\left(1-G\left(\beta^{\top} \boldsymbol{X} \mid \boldsymbol{X}\right)\right)-\left(1-F_{T \mid \boldsymbol{X}}\left(\beta^{\top} \boldsymbol{X} \mid \boldsymbol{X}\right)\right)\left(1-G_{C}\left(\beta^{\top} \boldsymbol{X} \mid \boldsymbol{X}\right)\right)\right\}\right],
\end{aligned}
$$

and observe that $M\left(\beta_{\tau}, G_{C}\right)=0$.

We now verify the conditions of Proposition 2 in Birke et al.. First, note that (C.1) trivially holds by construction of our estimator. Next, for $\beta \in \mathcal{B}_{\delta}$ let $\Gamma_{1}\left(\beta, G_{C}\right)$ denote the ordinary derivative of $M\left(\beta, G_{C}\right)$ with respect to $\beta$, that is,

$$
\begin{aligned}
\Gamma_{1}\left(\beta, G_{C}\right) & :=\frac{\partial M\left(\beta, G_{C}\right)}{\partial \beta} \\
& =\mathbb{E}_{\boldsymbol{X}}\left[\boldsymbol{X} \boldsymbol{X}^{\top}\left\{f_{T \mid \boldsymbol{X}}\left(\beta^{\top} \boldsymbol{X} \mid \boldsymbol{X}\right)\left(1-G_{C}\left(\beta^{\top} \boldsymbol{X} \mid \boldsymbol{X}\right)\right)+g_{C}\left(\beta^{\top} \boldsymbol{X} \mid \boldsymbol{X}\right)\left(\tau-F_{T \mid \boldsymbol{X}}\left(\beta^{\top} \boldsymbol{X} \mid \boldsymbol{X}\right)\right)\right\}\right],
\end{aligned}
$$

where $g_{C}(\cdot \mid \boldsymbol{x})$ denotes the density of $C$ conditionally on $\boldsymbol{X}=\boldsymbol{x}$. Under assumptions (C1)-(C4), $\Gamma_{1}\left(\beta, G_{C}\right)$ is then observed to be continuous and of full rank at $\beta_{\tau}$. Hence, condition (C.2) is satisfied in our framework. 
For condition (C.3), define first for all $\beta \in \mathcal{B}_{\delta}$ the functional derivative of $M(\beta, G)$ at $G_{C}$ in the direction $\left[G-G_{C}\right]$ as

$$
\begin{aligned}
\Gamma_{2}\left(\beta, G_{C}\right)\left[G-G_{C}\right] & :=\lim _{\eta \rightarrow 0} \frac{1}{\eta}\left[M\left(\beta, G_{C}+\eta\left(G-G_{C}\right)\right)-M\left(\beta, G_{C}\right)\right] \\
& =(1-\tau) \mathbb{E}_{\boldsymbol{X}}\left[\boldsymbol{X}\left(G_{C}\left(\beta^{\top} \boldsymbol{X} \mid \boldsymbol{X}\right)-G\left(\beta^{\top} \boldsymbol{X} \mid \boldsymbol{X}\right)\right)\right] .
\end{aligned}
$$

We then observe that for all $(\beta, G) \in \mathcal{B}_{\delta} \times \mathcal{G}_{\delta}, M(\beta, G)$ is linear in $G$ since $M(\beta, G)-M\left(\beta, G_{C}\right)-$ $\Gamma_{2}\left(\beta, G_{C}\right)\left[G-G_{C}\right]=0$. This verifies the first part of (C.3). For the second part, we have to show that $\left\|\Gamma_{2}\left(\beta, G_{C}\right)\left[\widehat{G}_{C}-G_{C}\right]-\Gamma_{2}\left(\beta_{\tau}, G_{C}\right)\left[\widehat{G}_{C}-G_{C}\right]\right\|=O_{\mathbb{P}}\left(n^{-1 / 2}\right)$. To prove this, using assumption (C5)-(ii), we have uniformly in $\beta \in \mathcal{B}_{\delta}$ that

$$
\Gamma_{2}\left(\beta, G_{C}\right)\left[\widehat{G}_{C}-G_{C}\right]=n^{-1} \sum_{i=1}^{n} \phi\left(Y_{i}, \Delta_{i}, \beta^{\top} \boldsymbol{X}_{i}, \boldsymbol{X}_{i}\right)+o \mathbb{P}\left(n^{-1 / 2}\right),
$$

where $\boldsymbol{\phi}\left(Y_{i}, \Delta_{i}, t, \boldsymbol{x}\right)=-(1-\tau) \boldsymbol{x} \xi\left(Y_{i}, \Delta_{i}, t \mid \boldsymbol{x}\right)$. An application of the central limit theorem then implies that $n^{1 / 2} \Gamma_{2}\left(\beta, G_{C}\right)\left[\widehat{G}_{C}-G_{C}\right] \stackrel{\mathcal{L}}{\rightarrow} \mathcal{N}(0, \boldsymbol{V})$ where $\boldsymbol{V}$ is finite under assumptions $(\mathrm{C} 1)$ and (C5)-(ii). This, in turn, implies that condition (C.3) in Birke et al. is satisfied in our context.

Next, condition (C.4) in Birke et al. is readily satisfied in our context by assumption (C5). To establish that condition (C.5) holds as well, we need to verify the conditions of Theorem 3 in Chen et al. (2003). To that end, let $m=m_{c}+m_{l c}$, where

$$
\begin{aligned}
m_{c}(Y, \boldsymbol{X}, \beta, G) & =\boldsymbol{X}(1-\tau)\left(1-G\left(\beta^{\top} \boldsymbol{X} \mid \boldsymbol{X}\right)\right) \\
m_{l c}(Y, \boldsymbol{X}, \beta, G) & =-\boldsymbol{X} \mathbb{1}\left(Y>\beta^{\top} \boldsymbol{X}\right) .
\end{aligned}
$$

Then, condition (3.1) is easily observed to hold for some $s_{1 j}, s_{j} \in(0,1]$ and $r=2$ under assumption (C1). For condition (3.2), as $m_{l c}$ does not depend here on $G$, we first note via the proof of Theorem 3 in Chen et al. that the constant $s_{j}$ controlling for the regularity of the nuisance parameter and appearing in condition (3.2) may in fact be replaced by the constant $s_{1 j}$ already appearing in condition (3.1). Therefore, we will verify condition (3.2) with respect to $s_{1 j}$ instead of $s_{j}$ as initially stated in Chen et al.. To that end, first it can be observed that for all positive values $\epsilon_{n}=o(1)$,

$$
\sup _{\beta_{\star}:\left\|\beta-\beta_{\star}\right\| \leq \epsilon_{n}}\left|\mathbb{1}\left(Y>\beta_{\star}^{\top} \boldsymbol{X}\right)-\mathbb{1}\left(Y>\beta^{\top} \boldsymbol{X}\right)\right| \leq \mathbb{1}\left(Y>\beta^{\top} \boldsymbol{X}-\epsilon_{n}\|\boldsymbol{X}\|\right)-\mathbb{1}\left(Y>\beta^{\top} \boldsymbol{X}+\epsilon_{n}\|\boldsymbol{X}\|\right) .
$$

Hence, we have that

$$
\begin{aligned}
& \mathbb{E}\left[\sup _{\beta_{\star}:\left\|\beta-\beta_{\star}\right\| \leq \epsilon_{n}}\left\|\boldsymbol{X}\left(\mathbb{1}\left(Y>\beta_{\star}^{\top} \boldsymbol{X}\right)-\mathbb{1}\left(Y>\beta^{\top} \boldsymbol{X}\right)\right)\right\|^{2}\right] \\
\leq & \mathbb{E}\left[\|\boldsymbol{X}\|^{2}\left\{\mathbb{1}\left(Y>\beta^{\top} \boldsymbol{X}-\epsilon_{n}\|\boldsymbol{X}\|\right)-\mathbb{1}\left(Y>\beta^{\top} \boldsymbol{X}+\epsilon_{n}\|\boldsymbol{X}\|\right)\right\}\right] \\
= & \mathbb{E}_{\boldsymbol{X}}\left[\|\boldsymbol{X}\|^{2}\left\{H\left(\beta^{\top} \boldsymbol{X}+\epsilon_{n}\|\boldsymbol{X}\| \mid \boldsymbol{X}\right)-H\left(\beta^{\top} \boldsymbol{X}-\epsilon_{n}\|\boldsymbol{X}\| \mid \boldsymbol{X}\right)\right\}\right] \\
\leq & \mathbb{E}_{\boldsymbol{X}}\left[\|\boldsymbol{X}\|^{3} K_{4} \epsilon_{n}\right],
\end{aligned}
$$

for some finite constant $K_{4}$ under assumptions (C2) and (C3). Hence, provided assumption (C1) is satisfied, we may observe that condition (3.2) holds for $s_{1 j}=1 / 2$. For the last condition of Theorem 3 in Chen et al., for $\epsilon>0$ denote first by $N\left(\epsilon, \mathcal{G}_{\delta},\|\cdot\|_{\mathcal{G}}\right)$ the covering number (Van der Vaart and Wellner $\left(1996\right.$, p. 83)) of the class $\mathcal{G}_{\delta}$ under the sup-norm metric we consider on the latter with a slight abuse of notation. Now, keeping in mind that $N\left(\epsilon, \mathcal{G}_{\delta},\|\cdot\|_{\mathcal{G}}\right) \leq N_{[]}\left(\epsilon, \mathcal{G}_{\delta},\|\cdot\|_{\mathcal{G}}\right)$, and since all the functions in the class $\mathcal{G}_{\delta}$ have values between 0 and 1 by $(\mathrm{C} 3)$, we first observe 
that only one $\epsilon$-bracket suffices to cover $\mathcal{G}_{\delta}$ if $\epsilon>1$. Then, using Lemma 6.1 in Lopez (2011) for a bound on the bracketing number for the case $\epsilon \leq 1$, we have that

$$
\begin{aligned}
\int_{0}^{\infty} \sqrt{\log N\left(\epsilon, \mathcal{G}_{\delta},\|\cdot\|_{\mathcal{G}}\right)} \mathrm{d} \epsilon & \leq \int_{0}^{1} \sqrt{\log N_{[]}\left(\epsilon, \mathcal{G}_{\delta},\|\cdot\|_{\mathcal{G}}\right)} \mathrm{d} \epsilon \\
& \leq K_{5} \int_{0}^{1} \epsilon^{-\frac{1}{1+\eta}} \mathrm{d} \epsilon \\
& <\infty
\end{aligned}
$$

for some finite constant $K_{5}$, hereby satisfying condition (3.3) in Chen et al. for $s_{j}=1$. It then follows from their Theorem 3 that condition (C.5) in Birke et al. holds in our context.

Lastly, for condition (C.6) we need to establish that $n^{1 / 2}\left[M_{n}\left(\beta_{\tau}, G_{C}\right)+\Gamma_{2}\left(\beta_{\tau}, G_{C}\right)\left[\widehat{G}_{C}-G_{C}\right]\right]$ converges to a normal distribution $\mathcal{N}(0, \Sigma)$ for some positive definite matrix $\Sigma$. Recalling that $M_{n}\left(\beta_{\tau}, G_{C}\right)=n^{-1} \sum_{i=1}^{n} m\left(Y_{i}, \boldsymbol{X}_{i}, \beta_{\tau}, G_{C}\right)$ is the average of independent random vectors with mean 0 , this follows easily using the same arguments as for the verification of condition (C.3) for the particular case of $\beta=\beta_{\tau}$. Hence, we obtain

$$
n^{1 / 2}\left[M_{n}\left(\beta_{\tau}, G_{C}\right)+\Gamma_{2}\left(\beta_{\tau}, G_{C}\right)\left[\widehat{G}_{C}-G_{C}\right]\right] \stackrel{\mathcal{L}}{\rightarrow} \mathcal{N}(0, \Sigma),
$$

where $\Sigma=\operatorname{Cov}\left(\Lambda_{i}\right)$ with

$$
\Lambda_{i}=m\left(Y_{i}, \boldsymbol{X}_{i}, \beta_{\tau}, G_{C}\right)-(1-\tau) \boldsymbol{X}_{i} \xi\left(Y_{i}, \Delta_{i}, \beta_{\tau}^{\top} \boldsymbol{X}_{i} \mid \boldsymbol{X}_{i}\right) .
$$

Theorem 3.2 then follows directly from an application of Proposition 2 in Birke et al., hereby concluding the proof. 


\section{Bibliography}

H. Bang and A. Tsiatis. Median regression with censored cost data. Biometrics, 58(3):643-649, 2002.

R. Beran. Nonparametric regression with randomly censored survival data. Technical report, Univ. California, Berkeley., 1981.

M. Birke, S. Van Bellegem, and I. Van Keilegom. Semi-parametric estimation in a single-index model with endogenous variables. Scandinavian Journal of Statistics, 44(1):168-191, 2017.

A. Bücher, A. El Ghouch, and I. Van Keilegom. Single-index quantile regression models for censored data. Submitted, 2014.

X. Chen, O. Linton, and I. Van Keilegom. Estimation of semiparametric models when the criterion function is not smooth. Econometrica, 71(5):1591-1608, 2003.

M. De Backer, A. El Ghouch, and I. Van Keilegom. Semiparametric copula quantile regression for complete or censored data. Electronic Journal of Statistics, 11(1):1660-1698, 2017.

L. Delsol and I. Van Keilegom. Semiparametric M-estimation with non-smooth criterion functions. Technical report, Université catholique de Louvain, 2015. URL http://www.uclouvain.be/ en-369695.html.

B. Efron. The two-sample problemn with censored data. Proceedings of the Fifth Berkeley Symposium in Mathematical Statistics, IV:831-853, 1967.

A. El Ghouch and I. Van Keilegom. Local linear quantile regression with dependent censored data. Statistica Sinica, 19:1621-1640, 2009.

J. B. Elsner, J. P. Kossin, and T. H. Jagger. The increasing intensity of the strongest tropical cyclones. Nature, 455(7209):92-95, 2008.

R. Gill. Large sample behaviour of the product-limit estimator on the whole line. The Annals of Statistics, pages 49-58, 1983.

W. Gonzalez-Manteiga and C. Cadarso-Suarez. Asymptotic properties of a generalized KaplanMeier estimator with some applications. Journal of Nonparametric Statistics, 4(1):65-78, 1994.

D. R. Hunter and K. Lange. Quantile regression via an MM algorithm. Journal of Computational and Graphical Statistics, 9(1):60-77, 2000.

J. Hyde. Testing survival with incomplete observations. Biostatistics Casebook, 1980.

R. Koenker. Quantile Regression. Cambridge Univ. Press., 2005.

R. Koenker and G. Jr. Bassett. Regression quantiles. Econometrica, 46(1):33-50, 1978.

R. Koenker and Y. Bilias. Quantile regression for duration data: A reappraisal of the pensylvania employment bonus experiments. Empirical Economics, 26:199-220, 2001. 
R. Koenker and O. Geling. Reappraising medfly longevity: a quantile regression survival analysis. J. Amer. Statist. Assoc., 96:458-468, 2001.

H. Koul, V. Susarla, and J. Van Ryzin. Regression analysis with randomly right-censored data. The Annals of Statistics, pages 1276-1288, 1981.

C. Leng and X. Tong. A quantile regression estimator for censored data. Bernoulli, 19(1):344-361, 2013.

A. Lindgren. Quantile regression with censored data using generalized L-1 minimization. Computational Statistics \& Data Analysis, 23(4):509-524, 1997.

S.-H. Lo and K. Singh. The product-limit estimator and the bootstrap: some asymptotic representations. Probability Theory and Related Fields, 71(3):455-465, 1986.

O. Lopez. Nonparametric estimation of the multivariate distribution function in a censored regression model with applications. Communications in Statistics-Theory and Methods, 40(15): 2639-2660, 2011.

L. Peng and Y. Huang. Survival analysis with quantile regression models. Journal of the American Statistical Association, 103(482):637-649, 2008.

S. Portnoy. Censored regression quantiles. J. Amer. Statist. Assoc., 98(464):1001-1012, 2003.

J.L. Powell. Least absolute deviations estimation for the censored regression model. Journal of Econometrics, 25(3):303-325, 1984.

J.L. Powell. Censored regression quantiles. Journal of Econometrics, 32:143-155, 1986.

J. H. Shows, W. Lu, and H. H. Zhang. Sparse estimation and inference for censored median regression. Journal of Statistical Planning and Inference, 140(7):1903-1917, 2010.

W. Stute. The oscillation behavior of empirical processes. The Annals of Probability, pages 86-107, 1982.

Y. Tang and H. Wang. Penalized regression across multiple quantiles under random censoring. Journal of Multivariate Analysis, 141:132-146, 2015.

A. W. Van der Vaart and J. A. Wellner. Weak Convergence and Empirical Processes. Springer, 1996.

I. Van Keilegom and M. G. Akritas. Transfer of tail information in censored regression models. Annals of Statistics, pages 1745-1784, 1999.

I. Van Keilegom and N. Veraverbeke. Estimation and bootstrap with censored data in fixed design nonparametric regression. Annals of the Institute of Statistical Mathematics, 49(3):467-491, 1997.

H. J. Wang and L. Wang. Locally weighted censored quantile regression. J. Amer. Statist. Assoc., 104:1117-1128, 2009.

H. J. Wang, J. Zhou, and Y. Li. Variable selection for censored quantile regresion. Statistica Sinica, 23(1):145-167, 2013.

A. Wey, L. Wang, and K. Rudser. Censored quantile regression with recursive partitioning-based weights. Biostatistics, 15(1):170-181, 2014.

Y. Wu and G. Yin. Multiple imputation for cure rate quantile regression with censored data. Biometrics, 2016. 
Z. Ying, S.H. Jung, and L.J. Wei. Survival analysis with median regression models. J. Amer. Statist. Assoc., 90:178-184, 1995.

L. Zhou. A simple censored median regression estimator. Statistica Sinica, pages 1043-1058, 2006. 